\title{
A SEQUENTIAL MINIMIZATION TECHNIQUE FOR ELLIPTIC QUASI-VARIATIONAL INEQUALITIES WITH GRADIENT CONSTRAINTS*
}

\author{
MICHAEL HINTERMÜLLER ${ }^{\dagger}$ AND CARLOS N. RAUTENBERG ${ }^{\ddagger}$
}

\begin{abstract}
A class of nonlinear elliptic quasi-variational inequality (QVI) problems with gradient constraints in function space is considered. Problems of this type arise, for instance, in the mathematical description of the magnetization of superconductors, in problems in elastoplasticity, or in electrostatics as well as in game theory. The paper addresses the iterative solution of the QVIs by a sequential minimization technique relying on the repeated solution of variational inequality-type problems. A monotone operator theoretic approach is developed which does not resort to Mosco convergence as is often done in connection with existence analysis for QVIs. For the numerical solution of the QVIs a penalty approach combined with a semismooth Newton iteration is proposed. The paper ends with a report on numerical tests involving the $p$-Laplace operator and various types of nonlinear constraints.
\end{abstract}

Key words. quasi-variational inequality, gradient constraints, sequential minimization, semismooth Newton method

AMS subject classifications. 47J20, 49J40, 49M15, 65J15, 65K10

DOI. $10.1137 / 110837048$

1. Introduction. Quasi-variational inequalities (QVIs), introduced by Bensoussan and Lions in $[9,32]$ to formulate impulse control problems, arise as mathematical models of various phenomena in the applied sciences. These involve areas such as, for instance, game theory, solid and continuum mechanics, or superconductivity. For related models and analytical investigations we refer the reader to, e.g., $[11,20,31,33,37,39]$, the monographs $[8,14,30]$, and the references therein. A recent study on stability issues for solutions of a rather general class of QVIs in finite dimensions can be found in [35].

While the feasible set of the QVIs may be rather general in finite dimensions (see, e.g., [35]), the situation for function space related problems is different, and the related literature is rather scarce. More specifically, most of the available work studies problems where the state (or solution) of the QVI has to satisfy pointwise constraints on a given subset of the underlying domain. This generalizes the variational inequality setting studied, e.g., in $[12,17,28,41]$ with respect to the choice of the involved bounds on the state. A particular instance of such a problem is the obstacle problem with a compliant obstacle [36].

In [39], Prigozhin developed a critical-state model for the stationary magnetization of superconductors. In this model the constitutive relation for the electric field

${ }^{*}$ Received by the editors June 10, 2011; accepted for publication (in revised form) June 20, 2012; published electronically October 9, 2012. This work was supported by the Austrian Ministry of Science and Education and the Austrian Science Fund FWF through START-Project Y305 "Interfaces and Free Boundaries," the German Research Foundation DFG Research Center MATHEON through Projects C28 and C31, and DFG Priority Program SPP 1253 through subproject "Elliptic Mathematical Programs with Equilibrium Constraints (MPECs) in Function Space: Optimality Conditions and Numerical Realization."

http://www.siam.org/journals/siopt/22-4/83704.html

${ }^{\dagger}$ Department of Mathematics, Humboldt-University of Berlin, 10099 Berlin, Germany (hint@ math.hu-berlin.de).

${ }^{\ddagger}$ Department of Mathematics and Scientific Computing, Karl-Franzens-University of Graz, A-8010 Graz, Austria (carlos.rautenberg@uni-graz.at). 
depends on the gradient of the vertical component of the associated magnetic field. This gives rise to a QVI which involves the $p$-Laplacian, a nonlinear elliptic partial differential operator, and pointwise constraints on the gradient of the associated state. An abstract generalization of the time dependent (parabolic) version of this problem was considered by Rodrigues and Santos in [42]. In the parabolic case, the solutions to this QVI approach solutions to the stationary model, i.e., the elliptic case, as time tends to infinity. The mathematical challenges in the elliptic as well as in the parabolic setting of this problem are due to the nonlinear nature of the involved differential operator and the general type of bounds in the gradient constraints.

Several other applications leading to QVIs with gradient constraints can be found in [31]. In fact, a class of elliptic QVIs with gradient constraints was analyzed by Kunze and Rodrigues in [31], where existence results (in some of the cases studied) follow from an application of Schauder's fixed-point theorem and convergence of the feasible sets in the sense of Mosco. Concerning the numerical treatment of this problem class, we note that the literature is extremely scarce. We also mention that compared to QVI problems with pointwise constraints on the state, problems containing pointwise gradient constraints are significantly more difficult to handle. This is due to the specific nonlinearity of the constraints, the nontrivial kernel of the gradient operator, and the associated analytical and numerical challenges.

In this paper we study an abstract version of the problem class considered in [31]. We establish a novel efficient sequential minimization technique for numerically approaching solutions to the QVI of interest that also proves existence. Our solution approach is based on monotone operator theory, an approximating sequence of variational inequalities (VIs), and semismooth Newton methods for solving the associated subproblems. In our report on the practical behavior of the overall algorithm we provide examples involving the $p$-Laplacian for $p=3$ and several types of gradient constraints.

The rest of this paper is organized as follows. In section 2 we state the class of QVI problems under investigation and its relation to a nonlinearly constrained minimization problem in function space. In section 3, we study a penalized version of the latter minimization problem, its asymptotic properties as the penalty parameter tends to infinity, and its relation to a specialized version of the QVI of interest. It turns out that a solution to the QVI solves the special QVI, which contains a possibly much smaller feasible set than the original QVI, but not vice versa. This obstacle is overcome by establishing Theorem 4.5 in section 4 , which provides a framework for an approximation method (related to a sequence of VIs) of solutions to the QVI. Continuity properties of the solutions to these VIs with respect to several parameters are studied as well. Based on our analytical findings, in section 5 we introduce an infinite dimensional algorithm for solving the QVI and provide details on its implementation. A penalty parameter update rule and a semismooth Newton solver (including convergence considerations) for the associated subproblems are the subjects of section 6 . This section ends with a report on numerical tests involving the $p$-Laplace operator. We provide differentiability properties (in infinite dimensions) for the $p$-Laplace and the penalty operators in Appendix A. Finally, in Appendix B, we establish conditions for which the solution mapping of a class of VIs is contractive with respect to the upper bound of the gradient constraint (addressing a hypothesis used in Theorem 4.5).

Notation. Throughout this paper, for a Banach space $X$ its norm is written as $|\cdot|_{X}$, with the exception of $\mathbb{R}^{l}$, where the (Euclidian) norm is written as $|\cdot|$. For 
$v_{0} \in X$ and $R>0$ we denote $B_{R}\left(v_{0}\right):=\left\{v \in X:\left|v-v_{0}\right|_{X}<R\right\}$ and its closure (in $X)$ by $\bar{B}_{R}\left(v_{0}\right)$. We denote the strong convergence of a sequence $\left\{u_{n}\right\} \subset X$ to $u \in X$ by $u_{n} \rightarrow u$. Weak convergence is written as $u_{n} \rightarrow u$. The Lebesgue measure of a measurable set $\Omega$ is denoted as $|\Omega|$, and we say that a property holds "a.e. (almost everywhere) in $\Omega$ " if it is true in $\Omega$ except for a subset $\Omega_{0} \subset \Omega$ such that $\left|\Omega_{0}\right|=0$. For a real-valued function $v$, we define $v^{+}=\max (0, v)$ in the pointwise sense, that is, $v^{+}=v$ if $v$ is nonnegative and zero otherwise.

2. Problem formulation. In this section we consider a class of QVI problems. A particular instance arises in the mathematical modeling of superconductors; see $[31,39,42]$. Let $\Omega \subset \mathbb{R}^{l}$ with $l \geq 2$ be a bounded and open domain, and define $W \equiv W_{0}^{1, p}(\Omega)$ for $1<p<\infty$ (see [1] for a definition of the Sobolev space $W_{0}^{1, p}(\Omega)$ ), with norm

$$
|v|_{W}=\left(\int_{\Omega}|\nabla v|^{p} \mathrm{~d} \mathbf{x}\right)^{1 / p}
$$

The (topological) dual space of $W$ is given by $W^{-1, p^{\prime}}(\Omega)$ with $p^{\prime}=p /(p-1)$ and is denoted by $W^{\prime}$. Also, $(\cdot, \cdot): W^{\prime} \times W \rightarrow \mathbb{R}$ defines the duality pairing between $W^{\prime}$ and $W$.

Suppose that $C$ is a closed and convex subset of $W$ such that $0 \in C$ and we are given a nonlinear mapping $\Phi: C \subset W \rightarrow L_{\nu}^{\infty}(\Omega) \subset L^{\infty}(\Omega)$, where $L_{\nu}^{\infty}(\Omega)=\{\varphi \in$ $L^{\infty}(\Omega): \varphi(\mathbf{x}) \geq \nu$ a.e. in $\left.\mathbf{x} \in \Omega\right\}$ with $\nu>0$. For $\varphi \in L_{\nu}^{\infty}(\Omega)$, the subset $K(\varphi)$ of $W$ defined as

$$
K(\varphi)=\{v \in W:|\nabla v(\mathbf{x})| \leq \varphi(\mathbf{x}) \text { a.e. on } \Omega\}
$$

is nonempty (since $0 \in K(\varphi)$ for any $\varphi \in L_{\nu}^{\infty}(\Omega)$ ), closed, and convex. Then, the same holds true for the subset $K(\Phi(u))$ of $W$ when $u \in C$ is fixed. The requirement " $\nu>0$ " is inherited from physical models in several applications (see, for example, $[31])$.

Suppose that $f \in W^{\prime}$ and $A: W \rightarrow W^{\prime}$. Then we define the problem $\left(\mathrm{P}_{\mathrm{QVI}}\right)$ as the following QVI:

$\left(\mathrm{P}_{\mathrm{QVI}}\right)$ Find $u \in C$ subject to $u \in K(\Phi(u)):(A(u)-f, v-u) \geq 0 \quad \forall v \in K(\Phi(u))$.

When $\Phi(u)=\varphi \in L_{\nu}^{\infty}(\Omega)$ for all $u \in C$, we refer to $\left(\mathrm{P}_{\mathrm{QVI}}\right)$ as $\left(\mathrm{P}_{\mathrm{VI}}\right)$ because the QVI reduces to a VI.

Unless otherwise stated, the nonlinear operator $A$ is assumed to satisfy the following hypotheses:

H1. It is uniformly monotone; i.e., there are constants $c>0$ and $r>1$ such that

$$
(A(u)-A(v), u-v) \geq c|u-v|_{W}^{r} \quad \forall u, v \in W .
$$

H2. It is continuous on finite dimensional spaces; i.e., if $M$ is a finite dimensional subspace of $W$, then $A: M \rightarrow W^{\prime}$ is weakly continuous; i.e., for each $v \in W$ the map $u \mapsto(A(u), v)$ is continuous on $M$.

H3. (Implied by H1) It is coercive, i.e., $(A(u), u) /|u|_{W} \rightarrow \infty$ as $|u|_{W} \rightarrow \infty$.

H4. (Implied by H2) It is hemicontinuous; i.e., the real-valued function $t \mapsto(A(u+t v), w)$ is continuous for $t \in[0,1]$ for all $u, v, w \in W$.

H5. It is homogeneous of degree $\beta>0$, i.e., $A(t u)=t^{\beta} A(u)$ for each $u \in W$ and $t>0$. 
H6. It is of potential type; i.e., there is a Gâteaux differentiable mapping $a: W \rightarrow$ $\mathbb{R}$ which is called the potential of $A$ such that

$$
\lim _{t \rightarrow 0} \frac{a(u+t v)-a(u)}{t}=(A(u), v) \quad \forall u, v \in W .
$$

H7. If $\left\{u_{n}\right\}$ is a sequence in $W$ such $u_{n} \rightarrow u$ in $W$ and $\left(A\left(u_{n}\right), u_{n}\right) \rightarrow(A(u), u)$ as $n \rightarrow \infty$, then $\left|\nabla u_{n}\right|_{L^{p}(\Omega)^{l}} \rightarrow|\nabla u|_{L^{p}(\Omega)^{l}}$ as $n \rightarrow \infty$.

H8. It is bounded in the following sense: There is a constant $r^{\prime}$ such that $0<$ $r^{\prime}<r$ (where $r>0$ is that of $\mathbf{H 1}$ ) and a nondecreasing continuous function $F:[0, \infty) \rightarrow[0, \infty)$ with $F(0)=0$ such that

$$
|(A(u), v)| \leq|u|_{W}^{r^{\prime}} F\left(|v|_{W}\right) \quad \forall u, v \in W .
$$

In some of the proofs ahead, $\mathbf{H} \mathbf{1}$ will be replaced by a weaker version: We say that $A$ is monotone if $u, v \in W$ implies $(A(u)-A(v), u-v) \geq 0$. We explicitly write $\mathbf{H 3}$ and $\mathbf{H} \mathbf{4}$ given the fact that we occasionally invoke these hypotheses without $\mathbf{H} \mathbf{1}$ or H2, respectively.

In [31], existence of solutions to $\left(\mathrm{P}_{\mathrm{QVI}}\right)$ is shown for $A: W \rightarrow W^{\prime}$ satisfying hypotheses H1, H2, and H8, with $\Phi: C \subset W \rightarrow L_{\nu}^{\infty}(\Omega)$ compact and continuous, where $C=\left\{v \in W:|v|_{W} \leq\left(|f|_{W^{\prime}} / c\right)^{1 /(r-1)}\right\}$.

If $A$ is monotone, homogeneous, hemicontinuous, and of potential type (hypotheses $\mathbf{H 1}, \mathbf{H 4}, \mathbf{H 5}$, and H6), then its potential $a: W \rightarrow \mathbb{R}$ (see [13]) is given by

$$
a(u)=\int_{0}^{1}(A(t u), u) \mathrm{d} t=(A(u), u) \int_{0}^{1} t^{\beta} \mathrm{d} t=\frac{(A(u), u)}{\beta+1} .
$$

The following is an important example of an operator that satisfies hypotheses H1H8.

Example 1. The $p$-Laplacian map $\Delta_{p}: W \rightarrow W^{\prime}$, defined as

$$
\left(\Delta_{p}(u), v\right):=\int_{\Omega}|\nabla u|^{p-2}(\nabla u \cdot \nabla v) \mathrm{d} \mathbf{x}
$$

for all $u, v \in W \equiv W_{0}^{1, p}(\Omega)$ and some $p \geq 2$, satisfies hypothesis $\mathbf{H 1 - H 8 . ~}$

Indeed, hypothesis $\mathbf{H} 1$ (and therefore $\mathbf{H 3}$ ) follows from the vector inequality $\left\langle|\mathbf{x}|^{p-2} \mathbf{x}-|\mathbf{y}|^{p-2} \mathbf{y}, \mathbf{x}-\mathbf{y}\right\rangle \geq c|\mathbf{x}-\mathbf{y}|^{p}$ (when $p \geq 2$ ) with some $c>0$ and $\mathbf{x}, \mathbf{y} \in \mathbb{R}^{l}$, which implies

$$
\left(\Delta_{p}(u)-\Delta_{p}(v), u-v\right) \geq c|u-v|_{W}^{p} .
$$

Continuity on finite dimensional subspaces (hypothesis $\mathbf{H 2}$ and therefore $\mathbf{H 4}$ ) follows from the study of differentiability of the map $u \mapsto \Delta_{p}(u)$ in Appendix A. Since $\Delta_{p}(t u)=t^{p-1} \Delta_{p}(u)$, the operator $\Delta_{p}$ is homogeneous of degree $p-1$ (hypothesis H5). An application of the Lebesgue bounded convergence theorem proves that $\Delta_{p}$ is of potential type (hypothesis $\mathbf{H 6}$ ) with the potential $a(u)=\frac{1}{p}\left(\Delta_{p}(u), u\right)=\frac{1}{p} \int_{\Omega}|\nabla u|^{p} \mathrm{~d} \mathbf{x}$ (for a proof, see [6]). Hypothesis $\mathbf{H 7}$ is verified by noting that $\left(\Delta_{p}(u), u\right)=\left|\nabla u_{n}\right|_{L^{p}(\Omega)^{l}}^{p}$. Finally, the operator $\Delta_{p}$ is bounded in the sense of hypothesis H8, i.e.,

$$
\left|\left(\Delta_{p}(u), v\right)\right| \leq \int_{\Omega}|\nabla u|^{p-1}|\nabla v| \mathrm{d} \mathbf{x} \leq|u|_{W}^{p-1}|v|_{W}
$$

Copyright (C) by SIAM. Unauthorized reproduction of this article is prohibited. 
While there is a rich literature on numerically solving $\left(\mathrm{P}_{\mathrm{VI}}\right)$, i.e., $\Phi(u) \equiv \varphi$ in $\left(\mathrm{P}_{\mathrm{QVI}}\right)$ (see $[29,25,18,23]$ and the references therein), the literature on QVI-solvers is relatively scarce, with $[19,42]$ being some of the very few references available. While in [19] a special problem structure is required, [42] rather offers a theoretical scheme which might be turned into a numerical algorithm. Typically, these approaches relate (sub)problems to specific minimization tasks. Based on this, below we relate $\left(\mathrm{P}_{\mathrm{QVI}}\right)$ to a particular minimization problem or even a sequence thereof. We start by introducing

$$
\left.\begin{array}{l}
\min J(u):=\frac{1}{\beta+1}(A(u), u)-(f, u) \text { over } u \in C \subset W \\
\text { subject to } u \in K(\Phi(u)),
\end{array}\right\}
$$

which we study next.

2.1. Relation between $(\mathbf{P})$ and $\left(\mathbf{P}_{\mathrm{QVI}}\right)$. Under certain hypotheses (for example, when $\Phi$ is constant and $C=W$, i.e., $\Phi(u)=\varphi \in L_{\nu}^{\infty}(\Omega)$ for all $\left.u \in W\right)$, $u^{*}$ is a solution to $\left(\mathrm{P}_{\mathrm{QVI}}\right)$ if and only if it is a solution to $(\mathrm{P})$ (for a proof, see [15] or [41]). In the case where $\Phi: C \subset W \rightarrow L_{\nu}^{\infty}(\Omega)$ is not constant and $C$ is a proper subset of $W$, this result does not hold in general (not even in finite dimensions; see [16]). A simple infinite dimensional counterexample is the following: Consider $p=2$ so that $W:=W_{0}^{1,2}(\Omega) \equiv H_{0}^{1}(\Omega)$ (a Hilbert space) with $\Omega:=(0,1) \times(0,1)$ and $A$ defined as $(A(u), v):=\int_{\Omega} \nabla u \cdot \nabla v \mathrm{~d} \mathbf{x}$ for all $u, v \in W$. It is straightforward to observe that $A$ satisfies hypotheses H1-H8. Let $C=\left\{u \in W:|u|_{W} \leq 1 / 2\right\}$ and $\Phi(u)=\nu$ if $u \in C$. Let also $v_{f} \in W$ such that $\left|v_{f}\right|_{W}=1$ and $\left|\nabla v_{f}(\mathbf{x})\right| \leq \nu$ a.e. in $\Omega$, and define $f \in W^{\prime}$ as $(f, v)=\int_{\Omega} \nabla v_{f} \cdot \nabla v \mathrm{~d} \mathbf{x}$ for all $v \in W$. In this case, Theorem 3.2 below shows that (P) has a solution. Alternatively, suppose $u^{*}$ solves $\left(\mathrm{P}_{\mathrm{QVI}}\right)$. Since $u^{*} \in C$ but $v_{f} \notin C$ (since $\left|v_{f}\right|_{W}=1$ ), then $\left|u^{*}-v_{f}\right|_{W}>0$; however, $v_{f} \in K\left(\Phi\left(u^{*}\right)\right)$. Therefore, choosing $v=v_{f}$ in $\left(\mathrm{P}_{\mathrm{QVI}}\right)$, we have

$$
\left(A\left(u^{*}\right)-f, v_{f}-u^{*}\right)=\int_{\Omega} \nabla\left(u^{*}-v_{f}\right) \cdot \nabla\left(v_{f}-u^{*}\right) \mathrm{d} \mathbf{x}=-\left|u^{*}-v_{f}\right|_{W}^{2} \geq 0,
$$

a contradiction.

Despite the previous fact, we prove in Theorem 4.5 that a solution to $\left(\mathrm{P}_{\mathrm{QVI}}\right)$ can be obtained (under additional hypotheses) as the limit of a sequence of solutions to problems of the type $(\mathrm{P})$.

We establish now a first relation between solutions to $\left(\mathrm{P}_{\mathrm{QVI}}\right)$ and $(\mathrm{P})$. For this purpose, we suppose that the operator $A: W \rightarrow W^{\prime}$ satisfies H1, H4, H5, and H6 (although $\mathbf{H 1}$ may be replaced by just monotonicity).

Proposition 2.1. If $u^{*}$ is a solution of $\left(\mathrm{P}_{\mathrm{QVI}}\right)$, it also solves

$$
\left.\begin{array}{l}
\min J(u):=\frac{1}{\beta+1}(A(u), u)-(f, u) \text { over } u \in W \\
\text { subject to } u \in K\left(\Phi\left(u^{*}\right)\right) .
\end{array}\right\}
$$

Alternatively, suppose $u^{*}$ is a solution to $(\mathrm{P})$ and $\Phi$ is concave, i.e., $\lambda \Phi\left(\psi_{1}\right)(\mathbf{x})+(1-$ $\lambda) \Phi\left(\psi_{2}\right)(\mathbf{x}) \leq \Phi\left(\lambda \psi_{1}+(1-\lambda) \psi_{2}\right)(\mathbf{x})$ for all $\psi_{1}, \psi_{2} \in C \subset W, \lambda \in[0,1]$, and $\mathbf{x} \in \Omega$. Then, $u^{*}$ satisfies

$$
u \in\left(K_{\Phi} \cap K(\Phi(u))\right):(A(u)-f, v-u) \geq 0 \quad \forall v \in\left(K_{\Phi} \cap K(\Phi(u))\right),
$$

where $K_{\Phi}=\{v \in C \subset W: v \in K(\Phi(v))\}$ (which is nonempty, since $0 \in K_{\Phi}$ ).

Before we prove Proposition 2.1, note that since $A$ satisfies hypotheses H1, H4, H5, and H6, its potential $a$ is given by $a(u)=(A(u), u) /(\beta+1)$. This implies that $u \mapsto J(u)$ is Gâteaux differentiable and $\left(J^{\prime}(u), v\right)=(A(u)-f, v)$, where $J^{\prime}(u) \in W^{\prime}$. 
Proof. Let $u^{*}$ be a solution of $\left(\mathrm{P}_{\mathrm{QVI}}\right)$, and let $w \in K\left(\Phi\left(u^{*}\right)\right)$ be arbitrary. Then $v:=(1-\lambda) u^{*}+\lambda w$ with $\lambda \in[0,1]$ belongs to $K\left(\Phi\left(u^{*}\right)\right)$ because the latter is a convex set and $u^{*} \in K\left(\Phi\left(u^{*}\right)\right)$. Then,

$$
\left(J^{\prime}\left(u^{*}\right), w-u^{*}\right)=\left(A\left(u^{*}\right)-f, w-u^{*}\right) \geq 0 \quad \forall w \in K\left(\Phi\left(u^{*}\right)\right),
$$

because $u^{*}$ solves ( $\mathrm{P}_{\mathrm{QVI}}$ ). Since $a^{\prime}=A$ is monotone, $a$ is convex (for a proof, see [46]). Henceforth $u \mapsto J(u)=a(u)-(f, u)$ is also convex. Therefore, $J\left((1-\lambda) u^{*}+\lambda w\right) \leq$ $(1-\lambda) J\left(u^{*}\right)+\lambda J(w)$, which implies that

$$
0 \leq\left(J^{\prime}\left(u^{*}\right), w-u^{*}\right)=\lim _{\lambda \downarrow 0} \frac{J\left(u^{*}+\lambda\left(w-u^{*}\right)\right)-J\left(u^{*}\right)}{\lambda} \leq J(w)-J\left(u^{*}\right)
$$

for all $w \in K\left(\Phi\left(u^{*}\right)\right)$, which proves the first claim.

Conversely, let $u^{*}$ be a solution to $(\mathrm{P})$, and let $w \in K_{\Phi} \cap K\left(\Phi\left(u^{*}\right)\right)$ be arbitrary with $\Phi$ concave. Then $v:=(1-\lambda) u^{*}+\lambda w$, with $\lambda \in[0,1]$, belongs to $K\left(\Phi\left((1-\lambda) u^{*}+\right.\right.$ $\lambda w))$; since $C$ is convex, $v \in C$ and $|\nabla v(\mathbf{x})| \leq(1-\lambda) \Phi\left(u^{*}\right)(\mathbf{x})+\lambda \Phi(w)(\mathbf{x}) \leq \Phi((1-$ $\left.\lambda) u^{*}+\lambda w\right)(\mathbf{x})$. Therefore, we have $J\left(u^{*}\right) \leq J\left(u^{*}+\lambda\left(w-u^{*}\right)\right)$, because $u^{*}$ is a solution to $(\mathrm{P})$, and the convexity of $J$ implies $J\left(u^{*}+\lambda\left(w-u^{*}\right)\right) \leq \lambda\left(J(w)-J\left(u^{*}\right)\right)+J\left(u^{*}\right)$. Hence,

$$
0 \leq\left(J^{\prime}\left(u^{*}\right), w-u^{*}\right)=\lim _{\lambda \downarrow 0} \frac{J\left(u^{*}+\lambda\left(w-u^{*}\right)\right)-J\left(u^{*}\right)}{\lambda} \leq J(w)-J\left(u^{*}\right) .
$$

Since $\left(J^{\prime}\left(u^{*}\right), w-u^{*}\right)=\left(A\left(u^{*}\right)-f, w-u^{*}\right)$ and $w \in K_{\Phi} \cap K\left(\Phi\left(u^{*}\right)\right)$ was arbitrary, the second claim is proved.

We note that for specific versions of the QVI such as for problems of finding Nash points in $n$-person games, there is a more direct characterization of the equivalence between solutions to a minimization problem of type $(\mathrm{P})$ and the QVI associated with it; see, for example, [8] or [10]. The reason for this relies on both the type of functionals being minimized and the structure of the convex sets that determine the constraints.

3. Penalty formulation. Problem $(\mathrm{P})$ poses several numerical and theoretical difficulties due to the constraint set $K(\Phi(\cdot)): W \rightarrow 2^{W}$. Most of these arise because of the nonlinearity of $\Phi$, or more specifically because $u \mapsto \Phi(u)$ may be nonsmooth and nonconcave (for example, in problems arising in superconductivity, operators of the form $\Phi(u)(\mathbf{x}):=\alpha(1+\beta|u(\mathbf{x})-h(\mathbf{x})|)^{-1}$ for some function $h$ and $\alpha, \beta>0$ are common). We approach this challenge by considering a sequence of penalized versions of problem $(\mathrm{P})$. The latter problems are important for Algorithm 1 and its convergence analysis and more specifically for step 2 of Algorithm 2. Moreover, a special version of these penalized problems is utilized in section 5 and subsequently in section 6 for the numerical solution of $\left(\mathrm{P}_{\mathrm{QVI}}\right)$.

A similar technique was recently used in PDE-constrained optimization [24] and for solving VIs of the first kind [18].

In this section we assume that $\Phi: C \subset W \rightarrow L_{\nu}^{\infty}(\Omega)$ is completely continuous (i.e., $\Phi$ maps weakly convergent sequences in $W$ into strongly convergent ones in $L^{\infty}(\Omega)$ ) and that $A: W \rightarrow W^{\prime}$ satisfies H1, H4, H5, H6, and H7 (although H1 may be relaxed by assuming only monotonicity of $A$ and $\mathbf{H 3}$ ).

For $\gamma \geq 0$, consider the penalized version of $(\mathrm{P})$ given by

$$
\min J_{\gamma}(u):=J(u)+\gamma J^{\mathrm{P}}(u) \text { over } u \in C \subset W
$$

Copyright (c) by SIAM. Unauthorized reproduction of this article is prohibited. 
with

$$
J(u):=\frac{1}{\beta+1}(A(u), u)-(f, u) \text { and } J^{\mathrm{P}}(u):=\frac{1}{2}\left|(|\nabla u|-\Phi(u))^{+}\right|_{L^{2}(\Omega)}^{2},
$$

where $(\cdot)^{+}$denotes $\max (0, \cdot)$ in the pointwise sense.

TheOREM 3.1. For each $\gamma \geq 0$, the problem $\left(\mathrm{P}_{\gamma}\right)$ admits at least one globally optimal solution.

Proof. Step 1: Existence of a weakly convergent infimizing sequence. Let $\left\{u_{n}\right\} \subset$ $C$ be an infimizing sequence of $J_{\gamma}$ in $W$, i.e., $\lim _{n \rightarrow \infty} J_{\gamma}\left(u_{n}\right)=\inf _{u \in C} J_{\gamma}(u)$. Note that $0 \in C$ and that $J_{\gamma}(0)=0$. For some $N \in \mathbb{N}$ and all $n \geq N$ we have that

$$
1=1+J_{\gamma}(0) \geq J_{\gamma}\left(u_{n}\right) \geq J\left(u_{n}\right) \geq\left(\frac{1}{\beta+1} \frac{\left(A\left(u_{n}\right), u_{n}\right)}{\left|u_{n}\right|_{W}}-|f|_{W^{*}}\right)\left|u_{n}\right|_{W},
$$

and hence $\left\{u_{n}\right\} \subset C$ is bounded in $W$; otherwise, $\frac{\left(A\left(u_{n}\right), u_{n}\right)}{\left|u_{n}\right| W} \rightarrow \infty$ for some subsequence of $\left\{u_{n}\right\}$ (because of the coercivity hypothesis in H3). Since $C$ is convex and closed, $\left\{u_{n}\right\}$ is uniformly bounded, and since $W$ is reflexive, there exist a subsequence of $\left\{u_{n}\right\}$ (also denoted by $\left\{u_{n}\right\}$ ) and $u^{*} \in C \subset W$ such that $u_{n} \rightarrow u^{*}$ in $W$.

Step 2: Weak lower semicontinuity of $u \mapsto J^{\mathrm{P}}(u)$. Since $u_{n} \rightarrow u^{*}$ in $W=$ $W_{0}^{1, p}(\Omega)$, we have $\nabla u_{n} \rightarrow \nabla u^{*}$ in $L^{p}(\Omega)$ and $\Phi\left(u_{n}\right) \rightarrow \Phi\left(u^{*}\right)$ in $L^{\infty}(\Omega)$ because $\Phi: C \subset W \rightarrow L_{\nu}^{\infty}(\Omega)$ is completely continuous.

Consider $g: \mathbb{R}^{l} \times \mathbb{R} \rightarrow \mathbb{R}$ defined as $g(\mathbf{s}, t)=\frac{1}{2}\left((|\mathbf{s}|-t)^{+}\right)^{2}$ which satisfies that $\mathbf{s} \mapsto$ $g(\mathbf{s}, t)$ is a convex function. Note that we may write $J^{\mathrm{P}}$ as $J^{\mathrm{P}}(u)=\int_{\Omega} g(\nabla u, \Phi(u)) \mathrm{d} \mathbf{x}$.

Let

$$
g\left(z_{n}, w_{n}\right)(\mathbf{x})=\frac{1}{2}\left(\left(\left|z_{n}(\mathbf{x})\right|-w_{n}(\mathbf{x})\right)^{+}\right)^{2}
$$

for all $\mathbf{x} \in \Omega$, where the sequences $\left\{z_{n}\right\}$ and $\left\{w_{n}\right\}$ satisfy $z_{n} \rightarrow z^{*}$ in $L^{p}(\Omega)$ and $w_{n} \rightarrow w^{*}$ in $L^{\infty}(\Omega)$.

If $z_{n} \rightarrow z^{*}$ in $L^{p}(\Omega)$, then there exist subsequences of $\left\{z_{n}\right\}$ and $\left\{w_{n}\right\}$ (also denoted by $\left\{z_{n}\right\}$ and $\left\{w_{n}\right\}$ ) which converge a.e. in $\Omega$ to $z^{*}$ and $w^{*}$. This in turn implies that $g\left(z_{n}, w_{n}\right)$ converges to $g\left(z^{*}, w^{*}\right)$ a.e. in $\Omega$, and then by Fatou's lemma,

$$
\liminf _{n \rightarrow \infty} \int_{\Omega} g\left(z_{n}, w_{n}\right) \mathrm{d} \mathbf{x} \geq \int_{\Omega} g\left(z^{*}, w^{*}\right) \mathrm{d} \mathbf{x} .
$$

Consider the case where $z_{n}$ does not converge strongly (but weakly) to $z^{*}$ in $L^{p}(\Omega)$. Let $M:=\liminf _{n \rightarrow \infty} \int_{\Omega} g\left(z_{n}, w_{n}\right) \mathrm{d} \mathbf{x}$. Then there exist subsequences of $\left\{z_{n}\right\}$ and $\left\{w_{n}\right\}$ (denoted by $\left\{z_{n_{i}}\right\}$ and $\left\{w_{n_{i}}\right\}$ ) such that $\lim _{i \rightarrow \infty} \int_{\Omega} g\left(z_{n_{i}}, w_{n_{i}}\right) \mathrm{d} \mathbf{x}=M$.

Suppose that $h_{1}$ and $h_{2}$ belong to $L^{2}(\Omega)$; then it follows that

$$
\left.|| h_{1}^{+}\right|_{L^{2}(\Omega)}-\left|h_{2}^{+}\right|_{L^{2}(\Omega)}|\leq| h_{1}^{+}-\left.h_{2}^{+}\right|_{L^{2}(\Omega)} \leq\left|h_{1}-h_{2}\right|_{L^{2}(\Omega)},
$$

and hence

$$
\begin{aligned}
\left|\left(\int_{\Omega} g\left(z_{n_{i}}, w^{*}\right) \mathrm{d} \mathbf{x}\right)^{1 / 2}-\left(\int_{\Omega} g\left(z_{n_{i}}, w_{n_{i}}\right) \mathrm{d} \mathbf{x}\right)^{1 / 2}\right| & \leq\left(\int_{\Omega}\left|w^{*}-w_{n_{i}}\right|^{2} \mathrm{~d} \mathbf{x}\right)^{1 / 2} \\
& \leq\left|w^{*}-w_{n_{i}}\right|_{L^{\infty}(\Omega)}|\Omega|^{1 / 2}
\end{aligned}
$$

Copyright $@$ by SIAM. Unauthorized reproduction of this article is prohibited. 
Then $\lim _{i \rightarrow \infty} \int_{\Omega} g\left(z_{n_{i}}, w^{*}\right) \mathrm{d} \mathbf{x}=M$ because $w_{n} \rightarrow w^{*}$ in $L^{\infty}(\Omega)$ by assumption. Therefore, for each $\epsilon>0$, there is an index $i_{\epsilon}$ such that for all $i \geq i_{\epsilon}$ it holds that

$$
\int_{\Omega} g\left(z_{n_{i}}, w^{*}\right) \mathrm{d} \mathbf{x} \leq M+\epsilon .
$$

Suppose $\epsilon>0$ is fixed. Since $z_{n} \rightarrow z^{*}$ in $L^{p}(\Omega)$, then by Mazur's lemma, there exists a sequence $\left\{\eta_{r}\right\}_{r=1}^{\infty} \subset$ co $\left\{z_{n_{i}}\right\}_{i=i_{\epsilon}}^{\infty}$ such that $\eta_{r} \rightarrow z^{*}$ in $L^{p}(\Omega)$, and for each $r \in \mathbb{N}$ there is an $R(r) \in \mathbb{N}$ such that

$$
\eta_{r}=\sum_{i=i_{\epsilon}}^{R(r)} \alpha_{i}^{r} z_{n_{i}}
$$

with $\sum_{i=i_{\epsilon}}^{R(r)} \alpha_{i}^{r}=1$ and $\alpha_{i}^{r} \geq 0$ for all $i_{\epsilon} \leq i \leq R(r)$ and all $r \in \mathbb{N}$. Here "co" denotes the convex hull. Since $\mathbf{s} \mapsto g(\mathbf{s}, t)$ is convex, we observe that

$$
\int_{\Omega} g\left(\eta_{r}, w^{*}\right) \mathrm{d} \mathbf{x} \leq \sum_{i=i_{\epsilon}}^{R(r)} \alpha_{i}^{r} \int_{\Omega} g\left(z_{n_{i}}, w^{*}\right) \mathrm{d} \mathbf{x} \leq M+\epsilon .
$$

By (3.1) and since $\eta_{r} \rightarrow z^{*}$, we know that $\int_{\Omega} g\left(z^{*}, w^{*}\right) \mathrm{d} \mathbf{x} \leq \liminf _{n \rightarrow \infty} \int_{\Omega} g\left(\eta_{r}, w^{*}\right) \mathrm{d} \mathbf{x}$, and by the definition of $M$ we have $M=\liminf _{n \rightarrow \infty} \int_{\Omega} g\left(z_{n}, w_{n}\right)$ dx , i.e.,

$$
\int_{\Omega} g\left(z^{*}, w^{*}\right) \mathrm{d} \mathbf{x} \leq \liminf _{n \rightarrow \infty} \int_{\Omega} g\left(z_{n}, w_{n}\right) \mathrm{d} \mathbf{x}+\epsilon .
$$

Since $\epsilon>0$ was arbitrary, we conclude $\int_{\Omega} g\left(z^{*}, w^{*}\right) \mathrm{d} \mathbf{x} \leq \liminf _{n \rightarrow \infty} \int_{\Omega} g\left(z_{n}, w_{n}\right) \mathrm{d} \mathbf{x}$. Let $z_{n}=\nabla u_{n}$ and $w_{n}=\Phi\left(u_{n}\right)$, and hence

$$
\int_{\Omega} g\left(\nabla u^{*}, \Phi\left(u^{*}\right)\right) \mathrm{d} \mathbf{x} \leq \liminf _{n \rightarrow \infty} \int_{\Omega} g\left(\nabla u_{n}, \Phi\left(u_{n}\right)\right) \mathrm{d} \mathbf{x} .
$$

This shows that $J^{\mathrm{P}}$ is weakly lower semicontinuous on $C \subset W=W_{0}^{1, p}(\Omega)$.

Step 3: Weak lower semicontinuity of $u \mapsto J(u)$. Since $A$ is monotone, homogeneous, hemicontinuous, and of potential type (hypotheses H1, H4, H5, and H6), its potential $a: W \rightarrow \mathbb{R}$ is given by $a(u)=(A(u), u) /(\beta+1)$ (see $(2.1)$ ). Its Gâteaux derivative $a^{\prime}=A$ is monotone, and then $a$ is convex. Also, $A$ is monotone and hemicontinuous, and $W$ is a real Banach space; therefore $A$ is demicontinuous. (A map $A: X \rightarrow X^{*}$, where $X$ is a Banach space, is called demicontinuous when $A\left(u_{n}\right) \rightarrow A(u)$ in the weak-star topology whenever $u_{n} \rightarrow u$ as $n \rightarrow \infty$. In our case, $X=W$ is reflexive, and hence the weak-star convergence is replaced by weak convergence, that is, $A\left(u_{n}\right) \rightarrow A(u)$ whenever $u_{n} \rightarrow u$ as $n \rightarrow \infty$. See, for example, [46].) Finally, since $W$ is reflexive, if $u_{n} \rightarrow u$, then $A\left(u_{n}\right) \rightarrow A(u)$ and $\left(A\left(u_{n}\right), u_{n}\right) \rightarrow(A(u), u)$, i.e., $u \mapsto a(u)=(A(u), u) /(\beta+1)$ is continuous. We have that $a$ is convex and continuous, and consequently it is weakly lower semicontinuous (see [46] for a proof). Therefore, $J(u)=(A(u), u) /(\beta+1)-(f, u)$ is weakly lower semicontinuous, and hence $J_{\gamma}=J+\gamma J^{\mathrm{P}}$ is weakly lower semicontinuous as well. Finally, $\inf _{u \in C} J_{\gamma}(u)=\lim _{n \rightarrow \infty} J_{\gamma}\left(u_{n}\right) \geq J_{\gamma}\left(u^{*}\right)$, which implies that $u^{*}$ is a global minimizer of $J_{\gamma}$ on $C \subset W$.

Remark. Note that the previous theorem also addresses the situation in which $\Phi$ is a constant operator, that is, $\Phi(u)=\varphi \in L_{\nu}^{\infty}(\Omega)$ for all $u \in C \subset W$. In this 
case, the existence of minimizers is obtained directly by observing the convexity of the functional $J_{\gamma}$. This same argument simplifies the previous proof in the case when $\Phi$ is concave (and then $-\Phi$ is convex).

Let $u_{\gamma}$ denote a solution of problem $\left(\mathrm{P}_{\gamma}\right)$. When $\Phi: W \rightarrow L_{\nu}^{\infty}(\Omega)$ is differentiable (we assume here that $C=W$ ), the optimality system (similarly obtained as in [24]) for this problem is given by

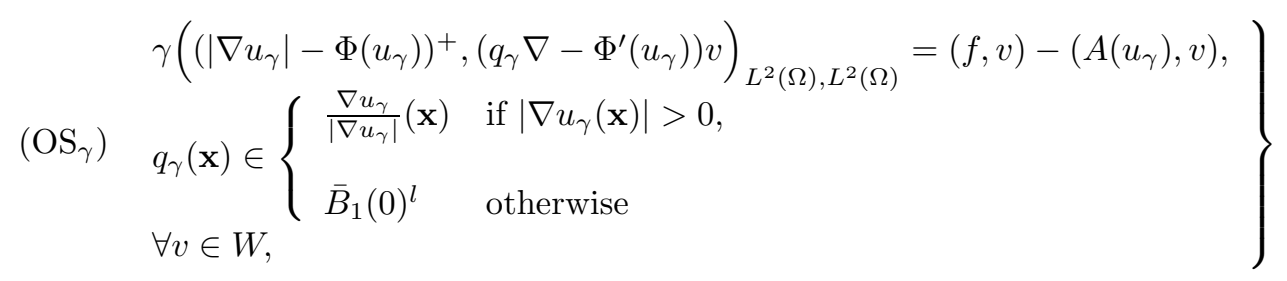

where $\bar{B}_{1}(0)^{l}$ denotes the closed unit ball in $\mathbb{R}^{l}$. Therefore, in the case of $\Phi(u)=\varphi \in$ $L_{\nu}^{\infty}(\Omega)$ for all $u \in W$, we have $\Phi^{\prime}(u)=0$, and the first equation of $\left(\operatorname{OS}_{\gamma}\right)$ reduces to

$$
\gamma\left(\left(\left|\nabla u_{\gamma}\right|-\varphi\right)^{+}, q_{\gamma} \nabla v\right)_{L^{2}(\Omega), L^{2}(\Omega)}=(f, v)-\left(A\left(u_{\gamma}\right), v\right) .
$$

The next theorem shows the asymptotic properties of the penalized problem $\left(\mathrm{P}_{\gamma}\right)$.

TheOREm 3.2. For each $\gamma \geq 0$, let $u_{\gamma} \in C \subset W$ denote a solution to the problem $\left(\mathrm{P}_{\gamma}\right)$. Then, for each sequence $\left\{\gamma_{n}\right\}$ of positive values tending to infinity, there is a subsequence $\left\{\gamma_{n_{k}}\right\}$ for which $u_{\gamma_{n_{k}}} \rightarrow \bar{u}$ in $W$ as $k \rightarrow \infty$, where $\bar{u} \in C$ is a solution to problem $(\mathrm{P})$.

Proof. Note that $0 \in C$ and $J_{\gamma}(0)=0$. Since $A$ satisfies the coercivity condition in $\mathbf{H 3}$, we observe that

$$
0=J_{\gamma}(0) \geq J_{\gamma_{n}}\left(u_{\gamma_{n}}\right) \geq\left(\frac{1}{\beta+1} \frac{\left(A\left(u_{\gamma_{n}}\right), u_{\gamma_{n}}\right)}{\left|u_{\gamma_{n}}\right|_{W}}-|f|_{W^{*}}\right)\left|u_{\gamma_{n}}\right|_{W}
$$

and then $\left\{u_{\gamma_{n}}\right\}$ is uniformly bounded in $W$. Since $C$ is convex and closed, $W$ is a reflexive Banach space, and $\left\{u_{\gamma_{n}}\right\}$ is uniformly bounded, there is a subsequence of $\left\{u_{\gamma_{n}}\right\}$ (also denoted by $\left\{u_{\gamma_{n}}\right\}$ ) such that $u_{\gamma_{n}} \rightarrow \bar{u}$ in $W$ when $\gamma_{n} \rightarrow \infty$ for some $\bar{u} \in C$.

Since $0=J_{\gamma_{n}}(0) \geq J_{\gamma_{n}}\left(u_{\gamma_{n}}\right)$ for all $\gamma_{n}$, it follows that $\gamma_{n} J^{\mathrm{P}}\left(u_{\gamma_{n}}\right)$ is uniformly bounded for all $\gamma_{n}$. Then, $J^{\mathrm{P}}\left(u_{\gamma_{n}}\right)=\left|\left(\left|\nabla u_{\gamma_{n}}\right|-\Phi\left(u_{\gamma_{n}}\right)\right)^{+}\right|_{L^{2}(\Omega)}^{2} \rightarrow 0$ as $\gamma_{n} \rightarrow \infty$. From $u_{\gamma_{n}} \rightarrow \bar{u}$ in $W$ as $\gamma_{n} \rightarrow \infty$, we observe that $0=\liminf _{n \rightarrow \infty} J^{\mathrm{P}}\left(u_{\gamma_{n}}\right) \geq J^{\mathrm{P}}(\bar{u}) \geq 0$ due to the weak lower semicontinuity of $J^{\mathrm{P}}$ (see the proof of Theorem 3.1). Therefore, $J^{\mathrm{P}}(\bar{u})=0$, which implies $|\nabla \bar{u}(\mathbf{x})| \leq \Phi(\bar{u})(\mathbf{x})$ for almost all $\mathbf{x} \in \Omega$, i.e.,

$$
\bar{u} \in K(\Phi(\bar{u})) .
$$

Let $\tilde{u} \in C \subset W$ with $\tilde{u} \in K(\Phi(\tilde{u}))$ be a solution to problem (P). Then, $J(\tilde{u}) \leq J(\bar{u})$ and $J_{\gamma_{n}}\left(u_{\gamma_{n}}\right) \leq J_{\gamma_{n}}(\tilde{u})$. Therefore, since $J$ is weakly lower semicontinuous,

$J(\bar{u}) \leq \liminf _{n \rightarrow \infty} J\left(u_{\gamma_{n}}\right) \leq \liminf _{n \rightarrow \infty} J_{\gamma_{n}}\left(u_{\gamma_{n}}\right) \leq \limsup _{n \rightarrow \infty} J_{\gamma_{n}}\left(u_{\gamma_{n}}\right) \leq \limsup _{n \rightarrow \infty} J_{\gamma_{n}}(\tilde{u})=J(\tilde{u})$,

which implies that $J(\bar{u})=J(\tilde{u})$ and $\lim _{n \rightarrow \infty} J_{\gamma_{n}}\left(u_{\gamma_{n}}\right)=J(\bar{u})$. Thus, $\bar{u} \in C$ is a global solution to problem $(\mathrm{P})$. 
Without loss of generality, suppose that $\gamma_{n+1} \geq \gamma_{n}$ for all $n \in \mathbb{N}$. Then, it is elementary to check that $J_{\gamma_{n+1}}\left(u_{\gamma_{n+1}}\right) \geq J_{\gamma_{n}}\left(u_{\gamma_{n}}\right)$ and $J(\bar{u}) \geq J_{\gamma_{n}}\left(u_{\gamma_{n}}\right)$ by

$$
J_{\gamma_{n+1}}\left(u_{\gamma_{n+1}}\right)=J\left(u_{\gamma_{n+1}}\right)+\gamma_{n+1} J^{\mathrm{P}}\left(u_{\gamma_{n+1}}\right) \geq J\left(u_{\gamma_{n+1}}\right)+\gamma_{n} J^{\mathrm{P}}\left(u_{\gamma_{n+1}}\right) \geq J_{\gamma_{n}}\left(u_{\gamma_{n}}\right),
$$

and $J(\bar{u})=J_{\gamma_{n}}(\bar{u}) \geq J_{\gamma_{n}}\left(u_{\gamma_{n}}\right)$. Hence, by standard optimization techniques for penalty functions (see, for example, [34, proof of Lemma 1, Section 10.11]) we observe that $\gamma_{n} J^{\mathrm{P}}\left(u_{\gamma_{n}}\right) \rightarrow 0$ as $n \rightarrow \infty$. From $\lim _{n \rightarrow \infty} J_{\gamma_{n}}\left(u_{\gamma_{n}}\right)=J(\bar{u})$ and $J_{\gamma_{n}}\left(u_{\gamma_{n}}\right)=$ $J\left(u_{\gamma_{n}}\right)+\gamma_{n} J^{\mathrm{P}}\left(u_{\gamma_{n}}\right)$, we have that $\lim _{n \rightarrow \infty} J\left(u_{\gamma_{n}}\right)=J(\bar{u})$. Note that $J(u)=(A(u), u) /$ $(\beta+1)-(f, u)$ and $\left(f, u_{\gamma_{n}}\right) \rightarrow(f, \bar{u})$ as $n \rightarrow \infty$; hence

$$
\lim _{n \rightarrow \infty}\left(A\left(u_{\gamma_{n}}\right), u_{\gamma_{n}}\right)=(A(\bar{u}), \bar{u}) .
$$

Since $u_{\gamma_{n}} \rightarrow \bar{u} \in C$ in $W=W_{0}^{1, p}(\Omega)$, then $\nabla u_{\gamma_{n}} \rightarrow \nabla \bar{u}$ in $L^{p}(\Omega)^{l}$, and also $u_{\gamma_{n}} \rightarrow \bar{u}$ in $L^{p}(\Omega)$ by the Rellich-Kondrakov theorem, which states that if $\Omega$ is a bounded domain of $\mathbb{R}^{l}$, then the canonical embedding $W_{0}^{1, p}(\Omega) \hookrightarrow L^{p}(\Omega)$ is compact (see, for example, [6]). Also, since $u_{\gamma_{n}} \rightarrow \bar{u}$ and $\left(A\left(u_{\gamma_{n}}\right), u_{\gamma_{n}}\right) \rightarrow(A(\bar{u}), \bar{u})$ as $n \rightarrow \infty$, then by $\mathbf{H 7}$ this implies that $\left|\nabla u_{n}\right|_{L^{p}(\Omega)^{l}} \rightarrow|\nabla u|_{L^{p}(\Omega)^{l}}$ as $n \rightarrow \infty$. This latter fact, together with $\nabla u_{\gamma_{n}} \rightarrow \nabla \bar{u}$ in $L^{p}(\Omega)$, implies that $\nabla u_{\gamma_{n}} \rightarrow \nabla \bar{u}$ in $L^{p}(\Omega)^{l}$, because of the locally uniform convexity of $L^{p}(\Omega)^{l}$ for $1<p<\infty$. Therefore, $u_{\gamma_{n}} \rightarrow \bar{u} \in C$ in $W=W_{0}^{1, p}(\Omega)$.

Remark. In the case of $\Phi(u)=\varphi \in L_{\nu}^{\infty}(\Omega)$ for all $u \in C=W$ and using the notation of the previous theorem, we have that $u_{\gamma_{n_{k}}}$ solves $\left(\mathrm{P}_{\gamma_{n_{k}}}\right)$, and its strong limit $\lim _{k \rightarrow \infty} u_{\gamma_{n_{k}}}=\bar{u}$ in turn solves $(\mathrm{P})$, which also solves $\left(\mathrm{P}_{\mathrm{QVI}}\right)$. For the case when $C$ is a proper subset of $W$ and $\Phi$ is nonconstant and completely continuous, we have that the strong limit of solutions of $\left(\mathrm{P}_{\gamma_{n_{k}}}\right), \lim _{k \rightarrow \infty} u_{\gamma_{n_{k}}}=\bar{u}$, solves (P). Moreover, if $\Phi$ is concave, then $\bar{u}$ satisfies

$$
u \in\left(K_{\Phi} \cap K(\Phi(u))\right):(A(u)-f, v-u) \geq 0 \quad \forall v \in\left(K_{\Phi} \cap K(\Phi(u))\right) ;
$$

cf. Proposition 2.1. This is a weaker result than saying that $u^{*}$ solves $\left(\mathrm{P}_{\mathrm{QVI}}\right)$; however, the following section (specifically Theorem 4.5) defines an iteration to approximate solutions to $\left(\mathrm{P}_{\mathrm{QVI}}\right)$, where each of the involved subproblems is a problem of type $(\mathrm{P})$.

4. Fixed-point-type iteration. Suppose $\varphi \in L_{\nu}^{\infty}(\Omega), f \in W^{\prime}$, and that $A$ : $W \rightarrow W^{\prime}$ satisfies hypotheses $\mathbf{H 1}, \mathbf{H 2}$, and H8. Let $S(A, f, \varphi)$ denote the unique solution (see [46] for a proof of uniqueness) to

$$
\text { find } u \in K(\varphi):(A(u)-f, v-u) \geq 0 \quad \forall v \in K(\varphi),
$$

where $K(\varphi)=\{v \in W:|\nabla v(\mathbf{x})| \leq \varphi(\mathbf{x})$ a.e. on $\mathbf{x} \in \Omega\}$. Using this notation, a solution to problem $\left(\mathrm{P}_{\mathrm{QVI}}\right)$ is given by $u=S(A, f, \Phi(u))$. Therefore, for a fixed $f \in W^{\prime}, \Phi: C \subset W \rightarrow L_{\nu}^{\infty}(\Omega)$, and $A: W \rightarrow W^{\prime}$, solutions to problem $\left(\mathrm{P}_{\mathrm{QVI}}\right)$ are given by fixed points of the mapping $u \mapsto S(A, f, \Phi(u))$ on $C \subset W$. The purpose of this section is to study the iteration $u_{n}=S\left(A_{n}, f_{n}, \Phi_{n}\left(u_{n-1}\right)\right)$, with $n \in \mathbb{N}$ and $u_{0} \in C \subset W$ given, for some sequences of monotone operators $\left\{A_{n}\right\}$ and of completely continuous mappings $\left\{\Phi_{n}\right\}$ (with the same domain $C \subset W$ ) and $\left\{f_{n}\right\} \subset W^{\prime}$. For analytical purposes, the continuity properties of the map $(A, f, \varphi) \mapsto S(A, f, \varphi)$ are studied first.

The following result establishes continuity (in some sense) of solutions $(A, f, \varphi) \mapsto$ $S(A, f, \varphi)$. For the proof we use an argument from [42, 43] and extend the result to our type of nonlinear monotone operators.

Copyright $@$ by SIAM. Unauthorized reproduction of this article is prohibited. 
Lemma 4.1. Let $f_{i} \in W^{\prime}$ with $\left|f_{i}\right|_{W^{\prime}} \leq c_{f}$ (for some constant $c_{f}>0$ ) and $\varphi_{i} \in L_{\nu}^{\infty}(\Omega)$ for $i=1,2$. Assume that $A_{1}, A_{2}: W \rightarrow W^{\prime}$ satisfy $\mathbf{H 1}$ (with the same constants $c>0$ and $r>1$ ), $\mathbf{H 2}$, and $\mathbf{H 8}$ (with the same $r^{\prime}>0$ such that $r^{\prime}<r$ and the same function $F)$. Define $u_{i}:=S\left(A_{i}, f_{i}, \varphi_{i}\right)$ for $i=1,2$. Then there are constants $C_{A}, C_{f}$, and $C_{\varphi}$ (depending only on $c_{f}$ ) such that

$$
\left|u_{1}-u_{2}\right|_{W}^{r} \leq C_{A} \delta_{R}\left(A_{2}, A_{1}\right)+C_{f}\left|f_{1}-f_{2}\right|_{W^{\prime}}+C_{\varphi}\left|\varphi_{1}-\varphi_{2}\right|_{L^{\infty}(\Omega)},
$$

where $\delta_{R}\left(A_{2}, A_{1}\right):=\sup _{v \in \bar{B}_{R}(0)}\left|A_{2}(v)-A_{1}(v)\right|_{W}$, and $\bar{B}_{R}(0)=\left\{v \in W:|v|_{W} \leq R\right\}$ with $R:=\left(c_{f} / c\right)^{\frac{1}{r-1}}$.

Proof. Define $\alpha:=1+\frac{\left|\varphi_{1}-\varphi_{2}\right|_{L}(\Omega)}{\nu}$, and notice that $\frac{\varphi_{1}(x)}{\alpha} \leq \varphi_{2}(x)$ and $\frac{\varphi_{2}(x)}{\alpha} \leq$ $\varphi_{1}(x)$ (recall that $\varphi_{i}(\mathbf{x}) \geq \nu>0$, since $\varphi_{i} \in L_{\nu}^{\infty}(\Omega)$ for $\left.i=1,2\right)$. Then, set $v_{1}:=\frac{u_{1}}{\alpha}$ and $v_{2}:=\frac{u_{2}}{\alpha}$, which satisfy $v_{1} \in K\left(\varphi_{2}\right)$ and $v_{2} \in K\left(\varphi_{1}\right)$ because of

$$
\left|\nabla v_{i}(x)\right| \leq \alpha^{-1}\left|\nabla u_{i}(x)\right| \leq \alpha^{-1} \varphi_{i}(x) \leq \varphi_{j}(x)
$$

for $(i, j)=(1,2),(2,1)$.

Using $v_{2}$ in (4.1), with $A=A_{1}, f=f_{1}$, and $u=u_{1}$, we observe that

$$
\left(A_{1}\left(u_{1}\right), u_{2}-u_{1}\right) \geq\left(f_{1}, u_{2}-u_{1}\right)+\left(1-\alpha^{-1}\right)\left(A_{1}\left(u_{1}\right)-f_{1}, u_{2}\right),
$$

and analogously, replacing $v_{1}$ in (4.1) with $A=A_{2}, f=f_{2}$, and $u=u_{2}$, we obtain the same inequality with " 1 " and "2" interchanged. Adding both inequalities and multiplying by -1 , we have that

$$
\begin{aligned}
& \left(A_{2}\left(u_{2}\right)-A_{1}\left(u_{1}\right), u_{2}-u_{1}\right) \\
& \quad \leq-\left(f_{1}-f_{2}, u_{2}-u_{1}\right)+\left(\alpha^{-1}-1\right)\left(\left(A_{1}\left(u_{1}\right)-f_{1}, u_{2}\right)+\left(A_{2}\left(u_{2}\right)-f_{2}, u_{1}\right)\right)
\end{aligned}
$$

or, equivalently,

$$
\begin{array}{rl}
\left(A_{2}\left(u_{2}\right)-A_{2}\left(u_{1}\right), u_{2}-u_{1}\right) \leq- & \left(A_{2}\left(u_{1}\right)-A_{1}\left(u_{1}\right)+f_{1}-f_{2}, u_{2}-u_{1}\right) \\
& +\left(\alpha^{-1}-1\right)\left(\left(A_{1}\left(u_{1}\right)-f_{1}, u_{2}\right)+\left(A_{2}\left(u_{2}\right)-f_{2}, u_{1}\right)\right) \\
=I & I I .
\end{array}
$$

We start by bounding $I$. Using $v=0$ in (4.1) and the uniform monotonicity (with the same $c>0$ and $r>1$ for $i=1,2)$ of the operators $A_{i}$, we have $\left|u_{i}\right|_{W} \leq$ $\left(\left|f_{i}\right|_{W^{\prime}} / c\right)^{\frac{1}{r-1}} \leq\left(c_{f} / c\right)^{\frac{1}{r-1}}=: R$ for $i=1,2$. Also, $|I| \leq\left(\left|A_{2}\left(u_{1}\right)-A_{1}\left(u_{1}\right)\right|_{W^{\prime}}+\mid f_{1}-\right.$ $\left.\left.f_{2}\right|_{W^{\prime}}\right)\left|u_{2}-u_{1}\right|_{W}$, and hence

$$
|I| \leq D_{1}\left(\delta_{R}\left(A_{2}, A_{1}\right)+\left|f_{1}-f_{2}\right|_{W^{\prime}}\right)
$$

with $D_{1}=2 R$ and $\bar{B}_{R}(0)=\left\{v \in W:|v|_{W} \leq R\right\}$.

We now turn our attention to $I I$. The boundedness condition $\mathbf{H 8}$ for $A_{i}$ implies that

$$
\left|\left(A_{1}\left(u_{1}\right)-f_{1}, u_{2}\right)+\left(A_{2}\left(u_{2}\right)-f_{2}, u_{1}\right)\right| \leq D_{2},
$$

where $D_{2}:=2\left(R^{r^{\prime}} F(R)+c_{f} R\right)$. Further, by the definition of $\alpha,\left|\frac{1}{\alpha}-1\right| \leq \frac{1}{\nu} \mid \varphi_{1}-$ $\left.\varphi_{2}\right|_{L^{\infty}(\Omega)}$, and then

$$
|I I| \leq\left(D_{2} / \nu\right)\left|\varphi_{1}-\varphi_{2}\right|_{L^{\infty}(\Omega)}
$$

Copyright (c) by SIAM. Unauthorized reproduction of this article is prohibited. 
Therefore, from (4.2), we have that

$$
\left(A_{2}\left(u_{2}\right)-A_{2}\left(u_{1}\right), u_{2}-u_{1}\right) \leq D_{1}\left(\delta_{R}\left(A_{2}, A_{1}\right)+\left|f_{1}-f_{2}\right|_{W^{\prime}}\right)+\left(D_{2} / \nu\right)\left|\varphi_{1}-\varphi_{2}\right|_{L^{\infty}(\Omega)} .
$$

Finally, the uniform monotonicity of $A_{2}$ (hypothesis H1) implies that

$$
\left|u_{1}-u_{2}\right|_{W}^{r} \leq\left(D_{1} / c\right)\left(\delta_{R}\left(A_{2}, A_{1}\right)+\left|f_{1}-f_{2}\right|_{W^{\prime}}\right)+\left(D_{2} / \nu c\right)\left|\varphi_{1}-\varphi_{2}\right|_{L^{\infty}(\Omega)} .
$$

Since $D_{1}$ and $D_{2}$ depend only on $c_{f}$, the claim is proved.

Remark 1. The result in Lemma 4.1 guarantees the continuity of the solution mapping $(f, \varphi) \mapsto S(A, f, \varphi)$ for a fixed $A$, but it does not guarantee Lipschitz continuity (which would be desirable later for the convergence of our numerical scheme) with respect to either of the variables $(f, \varphi)$. Under certain hypotheses, it can be proven that $f \mapsto S(A, f, \varphi)$ is Lipschitz continuous (see [43] or [41]) when $r=2$ in H1. Conditions for obtaining Lipschitz continuity (and the contraction property) of the map $\varphi \mapsto S(A, f, \varphi)$ are given in Theorem B.1. Suppose that $X$ and $Y$ are Banach spaces, and that $C_{X} \subset X$ is closed and convex. Consider the (generally nonlinear) map $\Phi: C_{X} \subset X \rightarrow Y$. We say that $\Phi$ is bounded if it maps bounded sets in $C_{X}$ into bounded sets in $Y$. If it maps bounded sets in $C_{X}$ into sets with compact closure in $Y$, then it is said that $\Phi$ is compact. Finally, if for every sequence $\left\{u_{n}\right\}$ such that $u_{n} \rightarrow u^{*}$ in $X$ we have $\Phi\left(u_{n}\right) \rightarrow \Phi\left(u^{*}\right)$, we say that $\Phi$ is completely continuous. Since $\Phi$ is allowed to be nonlinear, if $X$ and $Y$ are reflexive, and $\Phi$ is completely continuous, then it is compact, but the converse is not true in general. (For a counterexample consider $X=Y=L^{2}(I)$ with $I=(0,1), \Phi(u)=\int_{I}|u(t)|^{2} \mathrm{~d} t$. Then $\Phi$ is clearly compact because its range is one dimensional. Let $u_{n}(t)=\sin (\pi n t)$. Then $u_{n} \rightarrow 0$ in $X$ but $\Phi\left(u_{n}\right)=1 / 2 \neq 0=\Phi(0)$.) For convergence purposes, it is useful to define the following.

Definition 4.2. Suppose $X$ and $Y$ are Banach spaces. Let $\Phi, \Phi_{n}: C_{X} \subset X \rightarrow Y$ for $n \in \mathbb{N}$, where $C_{X}$ is closed. We say that the sequence $\left\{\Phi_{n}\right\}$ is continuously convergent to $\Phi$ (denoted as $\left.\Phi_{n} \stackrel{\mathrm{c}}{\longrightarrow} \Phi\right)$ if the following holds:

(a) For any bounded sequence $\left\{u_{n}\right\} \subset C_{X}$, the sequence $\left\{\Phi_{n}\left(u_{n}\right)\right\} \subset Y$ has a strongly convergent subsequence in $Y$.

(b) If $\left\{u_{n}\right\} \subset C_{X}$ and $u_{n} \rightarrow u^{*}$ in $X$, then $\Phi_{n}\left(u_{n}\right) \rightarrow \Phi\left(u^{*}\right)$ in $Y$ as $n \rightarrow \infty$.

If $\Phi_{n}=\Phi$ for all $n \in \mathbb{N}$, Definition 4.2 is equivalent to requiring that $\Phi$ be continuous and compact. However, in general, Definition 4.2 does not require $\Phi_{n}$, for $n \in \mathbb{N}$, to be continuous or compact: Let $X$ be an infinite dimensional Banach space and $C_{X}=X=Y$. Consider $\Phi_{n}(u)=\left(\operatorname{step}\left(1-|u|_{X}\right) / n\right) u$, where step $(t)=1$ if $t \geq 0$ and zero otherwise. It is straightforward to observe that $\Phi_{n} \stackrel{\text { c }}{\longrightarrow} 0$ and $\Phi_{n}$ is not continuous or compact for $n \in \mathbb{N}$.

In general, if $\Phi_{n} \stackrel{\text { c }}{\longrightarrow} \Phi$, then $\Phi$ is continuous. In fact, condition (b) of Definition 4.2 is sufficient for this (see, for example, $[4,3]$ ).

It is not clear whether $\Phi_{n} \stackrel{c}{\longrightarrow} \Phi$ implies that $\Phi$ is compact. However, if condition (a) of Definition 4.2 is strengthened by requiring that the set of cluster points for any subsequence of $\left\{\Phi_{n}\left(u_{n}\right)\right\} \subset Y$ (when $\left\{u_{n}\right\} \subset C$ is bounded) be nonempty, then this implies that $\Phi$ is compact. In this case, the set of all cluster points of $\left\{\Phi_{n}\left(u_{n}\right)\right\} \subset Y$, for all bounded sequences $\left\{u_{n}\right\} \subset C_{X}$, is compact (see [45] for a proof). Since the latter set contains $\overline{\Phi(B)}$ for any bounded $B \subset C_{X}$ (because of condition (b)), $\Phi$ is compact.

Sufficient conditions for satisfying (a) and (b) in Definition 4.2 are well known from the theory of collectively compact sets of operators (see [2]). For example, if 
$\left\{\Phi_{n}\right\}$ is collectively compact (i.e., $\overline{\cup_{n=1}^{\infty} \Phi_{n}(B)}$ is compact in $Y$ for any bounded set $B \subset C_{X}$ ), uniformly equicontinuous (i.e., for each $\epsilon>0$, there is a $\delta(\epsilon)>0$ such that $\left|\Phi_{n}(u)-\Phi_{n}(v)\right|_{Y}<\epsilon$ for $n \in \mathbb{N}$ if $|u-v|_{X}<\delta(\epsilon)$ with $\left.u, v \in C_{X}\right)$ and $\Phi$ lies in the pointwise closure of $\left\{\Phi_{n}\right\}$ (i.e., for any $u \in C_{X}, \Phi_{n}(u) \rightarrow \Phi(u)$ ), then conditions (a) and (b) in Definition 4.2 are satisfied. In this case, $\Phi$ is compact since the strengthened condition (a), described in the previous paragraph, is met.

Definition 4.3. Let $A, A_{n}: W \rightarrow W^{\prime}$ for $n \in \mathbb{N}$. We say that $\left\{A_{n}\right\}$ is uniformly convergent to $A: W \rightarrow W^{\prime}$ (denoted as $A_{n} \stackrel{\mathrm{u}}{\longrightarrow} A$ ) if the following hold:

(a) All $A_{n}$ satisfy $\mathbf{H 1}$ (with the same $c>0$ and same $r>1$ ), $\mathbf{H 2}$, and $\mathbf{H 8}$ (with the same $r^{\prime}>0$ such that $r^{\prime}<r$ and the same $F$ ).

(b) For each fixed $R>0$,

$$
\lim _{n \rightarrow \infty} \sup _{v \in \bar{B}_{R}(0)}\left|A_{n}(v)-A(v)\right|_{W^{\prime}}=0 .
$$

If $A_{n} \stackrel{\mathrm{u}}{\longrightarrow} A$ as $n \rightarrow \infty$, then $A$ satisfies $\mathbf{H 1}, \mathbf{H 2}$, and $\mathbf{H 8}$ (with the same parameters $c, r, r^{\prime}$, and $F$, as all $A_{n}$ ). If each $A_{n}$ is a linear continuous operator, condition (b) of Definition 4.3 is equivalent to requiring that $A \in \mathscr{L}\left(W, W^{\prime}\right)$ (the space of bounded linear operators from $W$ to $\left.W^{\prime}\right)$ and $\lim _{n \rightarrow \infty}\left|A_{n}-A\right|_{\mathscr{L}\left(W, W^{\prime}\right)}=0$.

Definition 4.4 (hypothesis A1). Let $f, f_{n} \in W^{\prime}, A, A_{n}: W \rightarrow W^{\prime}$, and $\Phi, \Phi_{n}$ : $C \subset W \rightarrow L_{\nu}^{\infty}(\Omega)$ for $n \in \mathbb{N}$ with $C$ closed and convex. We say that the sequence $u_{n}=S\left(A_{n}, f_{n}, \Phi_{n}\left(u_{n-1}\right)\right)$ with $n \in \mathbb{N}$ and $u_{0} \in C$ satisfies hypothesis $\mathrm{A} 1$ if

$$
\operatorname{Liminf}_{n \rightarrow \infty} S\left(A_{n}, f_{n}, \Phi_{n}\left(\overline{\mathrm{co}}\left\{u_{n}\right\}^{*}\right)\right) \subset \overline{\mathrm{co}} S\left(A, f, \Phi\left(\left\{u_{n}\right\}^{*}\right)\right),
$$

where Liminf denotes the lower limit in the sense of Kuratowski, $\left\{u_{n}\right\}^{*}$ denotes the set of cluster points of the sequence $\left\{u_{n}\right\}$, i.e., $\left\{u_{n}\right\}^{*}:=\left\{u \in W_{0}^{1, p}(\Omega): u_{n} \rightarrow u\right.$ for $n \in$ $\left.\mathbb{N}^{\prime} \subset \mathbb{N}\right\}$, and $\overline{\mathrm{co}}\left\{u_{n}\right\}^{*}$ denotes the closed convex hull of $\left\{u_{n}\right\}^{*}$.

We recall that if $\left\{M_{n}\right\}$ is a sequence of subsets of a Banach space $X$, then $\operatorname{Liminf}_{n \rightarrow \infty} M_{n}:=\left\{x \in X: \lim _{n \rightarrow \infty} d\left(x, M_{n}\right)=0\right\}$, where $d\left(x, M_{n}\right)=\inf _{y \in M_{n}} \mid x-$ $\left.y\right|_{X}($ see $[7])$.

Hypothesis A1 is simple to verify in certain situations, e.g., when $\left\{u_{n}\right\}^{*}$ is a singleton and $S\left(A_{n}, f_{n}, \Phi_{n}(v)\right) \rightarrow S(A, f, \Phi(v))$ as $n \rightarrow \infty$ for all $v \in C$. Although we will prove in Theorem 4.5 that the second condition always holds when $f_{n} \rightarrow f$ in $W^{\prime}, A_{n} \stackrel{\mathrm{u}}{\longrightarrow} A$, and $\Phi_{n} \stackrel{\mathrm{c}}{\longrightarrow} \Phi$, as $n \rightarrow \infty$, in order to prove that $\left\{u_{n}\right\}^{*}$ is a singleton, other assumptions are required. For example, consider that $A_{n}=A, f_{n}=f, \Phi_{n}=\Phi$ for all $n \in \mathbb{N}$. Suppose that for some closed, convex subset $M \subset W_{0}^{1, p}(\Omega)$, we observe that $S(A, f, \Phi(\cdot)): M \rightarrow M$ and that $u \mapsto S(A, f, \Phi(u))$ is contractive on $M$; that is, there is a $0<\alpha<1$ such that $\left|S\left(A, f, \Phi\left(u_{1}\right)\right)-S\left(A, f, \Phi\left(u_{2}\right)\right)\right|_{W} \leq \alpha\left|u_{1}-u_{2}\right|_{W}$ for all $u_{1}, u_{2} \in M$. Therefore, by Banach's contraction principle, the sequence $u_{n}=$ $S\left(A, f, \Phi\left(u_{n-1}\right)\right)$ for $n \in \mathbb{N}$ and $u_{0} \in M$ satisfies $\left\{u_{n}\right\}^{*}=\left\{u^{*}\right\}$ (a singleton). For the sake of completeness, in Appendix B we give conditions for $u \mapsto S(A, f, \Phi(u))$ to be contractive.

The following constitutes our main result. It proves the existence of solutions to $\left(\mathrm{P}_{\mathrm{QVI}}\right)$ and shows how to approach them by a sequence of solutions to VIs.

Theorem 4.5. Let $f, f_{n} \in W^{\prime}, A, A_{n}: W \rightarrow W^{\prime}$, and $\Phi, \Phi_{n}: C \subset W \rightarrow L_{\nu}^{\infty}(\Omega)$ for $n \in \mathbb{N}$ with $C$ closed and convex such that $C \supset \bar{B}_{R}(0)$ with $R=$ $\sup _{n \in \mathbb{N}}\left(\left|f_{n}\right|_{W^{\prime}} / c\right)^{1 /(r-1)}$ (where $r>1$ and $c>0$ are as in Definition 4.3). Suppose that $f_{n}$ is strongly convergent to $f \in W^{\prime}, \Phi_{n}$ is continuously convergent to a compact $\Phi$ (Definition 4.2), and that $A_{n}$ is uniformly convergent to $A$ (Definition 
4.3), i.e.,

$$
\begin{gathered}
A_{n} \stackrel{\mathrm{u}}{\longrightarrow} A, \quad \Phi_{n} \stackrel{\mathrm{c}}{\longrightarrow} \Phi, \quad \text { and } f_{n} \rightarrow f \text { in } W^{\prime}, \\
\text { as } \left.n \rightarrow \vec{A}_{n}, f_{n}, \Phi_{n}\left(u_{n-1}\right)\right) ; \text { i.e., } u_{n} \in W \text { is the unique solution to } \\
u_{n}=S\left(u_{n}\right\}_{n=0}^{\infty} \text { defined as }
\end{gathered}
$$

$$
\text { find } u \in K\left(\Phi_{n}\left(u_{n-1}\right)\right):\left(A_{n}(u)-f_{n}, v-u\right) \geq 0 \quad \forall v \in K\left(\Phi_{n}\left(u_{n-1}\right)\right) .
$$

Suppose that $\left\{u_{n}\right\}$ satisfies hypothesis A1.

Then, there is a $u^{*} \in \overline{\operatorname{co}}\left\{u_{n}\right\}^{*}$, where $u^{*}=S\left(A, f, \Phi\left(u^{*}\right)\right)$; i.e., $u^{*}$ solves

$$
\text { find } u \in K(\Phi(u)):(A(u)-f, v-u) \geq 0 \quad \forall v \in K(\Phi(u)) \text {. }
$$

If $\left\{u_{n}\right\}^{*}=\left\{u^{*}\right\}$ (a singleton), $u_{n} \rightarrow u^{*}$ in $W$ (along a subsequence), where $u^{*}=$ $S\left(A, f, \Phi\left(u^{*}\right)\right)$.

Proof. Step 1: The set $\left\{u_{n}\right\}^{*}$ is not empty. Using $v=0$ in the VI given in (4.3) and because the operators $A_{n}$ satisfy hypothesis H1 (with uniform $c$ and $r$ for all $n \in \mathbb{N})$, we have $\left|u_{n}\right|_{W} \leq\left(\left|f_{n}\right|_{W^{\prime}} / c\right)^{\frac{1}{r-1}}$. Then, the sequence $\left\{\Phi_{n}\left(u_{n-1}\right)\right\}$ is well defined, because $\left\{u_{n}\right\} \subset \bar{B}_{R}(0) \subset C$ with $R=\sup _{n \in \mathbb{N}}\left(\left|f_{n}\right|_{W^{\prime}} / c\right)^{\frac{1}{r-1}}$. Since $\Phi_{n} \stackrel{c}{\longrightarrow} \Phi$, condition (a) in Definition 4.2 implies that $\Phi_{n}\left(u_{n-1}\right) \rightarrow y$ in $L^{\infty}(\Omega)$ for some $y$ when $n \in \mathbb{N}^{\prime} \subset \mathbb{N}$. Lemma 4.1 states that

$$
\left|u_{n}-u_{m}\right|_{W}^{r} \leq C_{A} \delta_{R}\left(A_{n}, A_{m}\right)+C_{f}\left|f_{n}-f_{m}\right|_{W^{\prime}}+C_{\varphi}\left|\Phi_{n}\left(u_{n-1}\right)-\Phi_{m}\left(u_{m-1}\right)\right|_{L^{\infty}(\Omega)} .
$$

We know that $\left\{\Phi_{n}\left(u_{n-1}\right)\right\}$ with $n \in \mathbb{N}^{\prime} \subset \mathbb{N}$ is a Cauchy sequence in $L^{\infty}(\Omega)$ and $A_{n} \stackrel{\mathrm{u}}{\longrightarrow} A$ implies $\delta_{R}\left(A_{n}, A_{m}\right) \rightarrow 0$, as $m, n \rightarrow \infty$. Also, we have that $f_{n} \rightarrow f$ in $W^{\prime}$. Therefore $\left\{u_{n}\right\}$ with $n \in \mathbb{N}^{\prime} \subset \mathbb{N}$ is a Cauchy sequence in $W$ and has a strong limit. Thus, $u_{n} \rightarrow u^{*}$ for $n \in \mathbb{N}^{\prime} \subset \mathbb{N}$ in $W$ for some $u^{*} \subset W$, and since $u_{n} \in C$ for $n \in \mathbb{N}^{\prime} \subset \mathbb{N}$, then $u^{*} \in C$. Hence, $\left\{u_{n}\right\}^{*}$ is not empty.

Step 2: If $w_{n} \rightarrow w^{*}$ in $W$, then $S\left(A_{n}, f_{n}, \Phi_{n}\left(w_{n}\right)\right) \rightarrow S\left(A, f, \Phi\left(w^{*}\right)\right)$ in $W$ as $n \rightarrow \infty$. Let $y_{n}:=S\left(A_{n}, f_{n}, \Phi_{n}\left(w_{n}\right)\right)$. We know that $\Phi_{n}\left(w_{n}\right) \rightarrow \Phi\left(w^{*}\right)$ in $L^{\infty}(\Omega)$ (because of condition (b) in Definition 4.2). Then, by Lemma 4.1, we observe that $\left\{y_{n}\right\}$ is a Cauchy sequence in $W$, and then $y_{n} \rightarrow y^{*}$ in $W$, for some $y^{*} \in W$ as $n \rightarrow \infty$. The strong convergence (in $W=W_{0}^{1, p}(\Omega)$ ) of $y_{n}$ to $y^{*}$ implies the strong convergence of $\nabla y_{n}$ to $\nabla y^{*}$ in $L^{p}(\Omega)^{l}$. For a subsequence of $\left\{y_{n}\right\}$ (denoted also by $\left.\left\{y_{n}\right\}\right), \nabla y_{n}(\mathbf{x}) \rightarrow \nabla y^{*}(\mathbf{x})$ for almost all $\mathbf{x} \in \Omega$. Hence, $\left|\nabla y_{n}(\mathbf{x})\right| \leq \Phi_{n}\left(w_{n}(\mathbf{x})\right)$ a.e. (because $y_{n}=S\left(A_{n}, f_{n}, \Phi_{n}\left(w_{n}\right)\right)$ ) implies that $\left|\nabla y^{*}(x)\right| \leq \Phi\left(w^{*}(x)\right)$ for almost all $\mathbf{x} \in \Omega$, i.e., $y^{*} \in K\left(\Phi\left(w^{*}\right)\right)$.

Step 2.1: For arbitrary $v \in K\left(\Phi\left(w^{*}\right)\right)$, there is a sequence $\left\{v_{k}\right\}$ such that $v_{k} \in$ $K\left(\Phi_{n_{k}}\left(w_{n_{k}}\right)\right)$ with $k=1,2, \ldots$ for some subsequence $\left\{w_{n_{k}}\right\}$ of $\left\{w_{n}\right\}$ and $v_{k} \rightarrow v$ in $W$. Since $\Phi\left(w^{*}\right), \Phi_{n}\left(w_{n}\right) \in L_{\nu}^{\infty}(\Omega)$, we have that $\Phi\left(w^{*}\right)(\mathbf{x}) \geq \nu$ and $\Phi_{n}\left(w_{n}\right)(\mathbf{x}) \geq \nu$ for almost all $\mathbf{x} \in \Omega$ and for all $n=1,2, \ldots$. Also, $\Phi_{n}\left(w_{n}\right) \rightarrow \Phi\left(w^{*}\right)$ in $L^{\infty}(\Omega)$. Therefore we next show that for any $\eta \in(0,1)$ there is an $N(\eta)$ such that

$$
0 \leq \eta \Phi\left(w^{*}\right)(\mathbf{x}) \leq \Phi_{n}\left(w_{n}\right)(\mathbf{x})
$$

for almost all $\mathbf{x} \in \Omega$ and for all $n \geq N(\eta)$. Indeed, let $\eta \in(0,1)$ be arbitrary and $\Omega_{n}:=\left\{\mathbf{x} \in \Omega: \eta \Phi\left(w^{*}\right)(\mathbf{x})>\Phi_{n}\left(w_{n}\right)(\mathbf{x})\right\}$. Then, for almost all $\mathbf{x} \in \Omega_{n}$, we observe that

$\left|\Phi\left(w^{*}\right)-\Phi_{n}\left(w_{n}\right)\right|_{L^{\infty}(\Omega)} \geq \Phi\left(w^{*}\right)(\mathbf{x})-\Phi_{n}\left(w_{n}\right)(\mathbf{x})>(1-\eta) \Phi\left(w^{*}\right)(\mathbf{x}) \geq(1-\eta) \nu>0$. 
But $\left|\Phi\left(w^{*}\right)-\Phi_{n}\left(w_{n}\right)\right|_{L^{\infty}(\Omega)} \rightarrow 0$, and hence for some large enough $N(\eta)$, we have that $\left|\Omega_{n}\right|=0$ for all $n \geq N(\eta)$.

Now, let $\left\{\eta_{n}\right\}$ be a monotonically increasing sequence in $(0,1)$ such that $\lim _{n \rightarrow \infty} \eta_{n}=1$, and let $v \in K\left(\Phi\left(w^{*}\right)\right)$ be arbitrary. Hence, the sequence $\left\{v_{n}\right\}$ with $v_{n}=\eta_{n} v$ belongs to $K\left(\Phi_{N\left(\eta_{n}\right)}\left(w_{N\left(\eta_{n}\right)}\right)\right)$, i.e.,

$$
\left|\nabla v_{n}(\mathbf{x})\right|=\left|\eta_{n} \nabla v(\mathbf{x})\right| \leq \eta_{n} \Phi\left(w^{*}\right)(\mathbf{x}) \leq \Phi_{N\left(\eta_{n}\right)}\left(w_{N\left(\eta_{n}\right)}\right)(\mathbf{x})
$$

for almost all $\mathbf{x} \in \Omega$. Also, we have $\left|v-v_{n}\right|_{W}=\left(1-\eta_{n}\right)|v|_{W}$, i.e., $v_{n} \rightarrow v$ in $W$.

Step 2.2: $y^{*}=S\left(A, f, \Phi\left(w^{*}\right)\right)$. The operator $A$ is monotone and hemicontinuous, and $W$ is a real Banach space. Therefore $A$ is demicontinuous (for a proof, see [46]); i.e., from $y_{n} \rightarrow y^{*}$ in $W$, we infer that $A\left(y_{n}\right) \rightarrow A\left(y^{*}\right)$ in $W^{\prime}$. Since $A_{n}\left(y_{n}\right)=$ $\left(A_{n}\left(y_{n}\right)-A\left(y_{n}\right)\right)+A\left(y_{n}\right)$, and $\left|A_{n}\left(y_{n}\right)-A\left(y_{n}\right)\right|_{W^{\prime}} \leq \delta_{R}\left(A_{n}, A\right) \rightarrow 0$ as $n \rightarrow \infty$, we have that $A_{n}\left(y_{n}\right) \rightarrow A\left(y^{*}\right)$ in $W^{\prime}$.

Finally, we have that $\left(A_{N\left(\eta_{n}\right)}\left(y_{N\left(\eta_{n}\right)}\right)-f_{N\left(\eta_{n}\right)}\right) \rightarrow\left(A\left(y^{*}\right)-f\right)$ in $W^{\prime}$ and $\left(v_{n}-y_{N\left(\eta_{n}\right)}\right) \rightarrow\left(v-y^{*}\right)$ in $W$, and hence

$$
\left(A_{N\left(\eta_{n}\right)}\left(y_{N\left(\eta_{n}\right)}\right)-f_{N\left(\eta_{n}\right)}, v_{n}-y_{N\left(\eta_{n}\right)}\right) \longrightarrow\left(A\left(y^{*}\right)-f, v-y^{*}\right),
$$

as $n \rightarrow \infty$. Since $\left(A\left(y_{N\left(\eta_{n}\right)}\right)-f_{N\left(\eta_{n}\right)}, v_{n}-y_{N\left(\eta_{n}\right)}\right) \geq 0$ and $v \in K\left(\Phi\left(w^{*}\right)\right)$ is arbitrary, we observe that

$$
\left(A\left(y^{*}\right)-f, v-y^{*}\right) \geq 0
$$

for all $v \in K\left(\Phi\left(w^{*}\right)\right)$, i.e., $y^{*}$ is identical to $S\left(A, f, \Phi\left(w^{*}\right)\right)$, which implies that $S\left(A_{n}, f_{n}, \Phi_{n}\left(w_{n}\right)\right) \rightarrow S\left(A, f, \Phi\left(w^{*}\right)\right)$ in $W$ if $w_{n} \rightarrow w^{*}$ in $W$, as $n \rightarrow \infty$.

Step 3: There is a $u^{*} \in \overline{\mathrm{co}}\left\{u_{n}\right\}^{*}$ such that $u^{*}=S\left(A, f, \Phi\left(u^{*}\right)\right)$. Let $\bar{u} \in\left\{u_{n}\right\}^{*}$ be arbitrary. Then, $u_{n_{j}} \rightarrow \bar{u}$ as $j \rightarrow \infty$ (for some subsequence $\left\{u_{n_{j}}\right\}$ of $\left\{u_{n}\right\}$ ), and hence $u_{n_{j}+1}=S\left(A_{n_{j}+1}, f_{n_{j}+1}, \Phi_{n_{j}+1}\left(u_{n_{j}}\right)\right) \rightarrow S(A, f, \Phi(\bar{u}))=\tilde{u} \in\left\{u_{n}\right\}^{*}$ as $j \rightarrow \infty$. Therefore, $S\left(f, A, \Phi\left(\left\{u_{n}\right\}^{*}\right)\right) \subset\left\{u_{n}\right\}^{*}$, and this implies that $\overline{c o} S\left(f, A, \Phi\left(\left\{u_{n}\right\}^{*}\right)\right) \subset$ $\overline{\mathrm{co}}\left\{u_{n}\right\}^{*}$.

Since the sequence $u_{n}=S\left(A_{n}, f_{n}, \Phi_{n}\left(u_{n-1}\right)\right)$ for $n \in \mathbb{N}$ and $u_{0} \in C$ satisfies hypothesis A1, we have that $\operatorname{Liminf}_{n \rightarrow \infty} S\left(A_{n}, f_{n}, \Phi_{n}\left(\overline{\mathrm{co}}\left\{u_{n}\right\}^{*}\right)\right) \subset \overline{\mathrm{co}} S\left(A, f, \Phi\left(\left\{u_{n}\right\}^{*}\right)\right)$. Also, for a fixed $\hat{u} \in \overline{\mathrm{Co}}\left\{u_{n}\right\}^{*}$, we have that $S\left(A_{n}, f_{n}, \Phi_{n}(\hat{u})\right) \rightarrow S(A, f, \Phi(\hat{u}))$. Therefore, $S\left(A, f, \Phi\left(\overline{\mathrm{co}}\left\{u_{n}\right\}^{*}\right)\right) \subset \operatorname{Liminf}_{n \rightarrow \infty} S\left(A_{n}, f_{n}, \Phi_{n}\left(\overline{\mathrm{co}}\left\{u_{n}\right\}^{*}\right)\right)$. This implies that

$$
S\left(A, f, \Phi\left(\overline{\operatorname{co}}\left\{u_{n}\right\}^{*}\right)\right) \subset \overline{\operatorname{co}} S\left(A, f, \Phi\left(\left\{u_{n}\right\}^{*}\right)\right) \subset \overline{\operatorname{co}}\left\{u_{n}\right\}^{*},
$$

i.e., $S(A, f, \Phi(\cdot)): \overline{\mathrm{co}}\left\{u_{n}\right\}^{*} \rightarrow \overline{\mathrm{co}}\left\{u_{n}\right\}^{*}$. Since $\varphi \mapsto S(A, f, \varphi)$ is continuous (Lemma 4.1 ), and $u \mapsto \Phi(u)$ is continuous (because $\Phi_{n} \stackrel{\text { c }}{\longrightarrow} \Phi$ ) and compact, then $u \mapsto$ $S(A, f, \Phi(u))$ is continuous and compact, and since $\overline{\mathrm{co}}\left\{u_{n}\right\}^{*}$ is closed, bounded, and convex, by Schauder's fixed-point theorem, there is a $u^{*} \in \overline{\mathrm{co}}\left\{u_{n}\right\}^{*}$ such that $u^{*}=$ $S\left(A, f, \Phi\left(u^{*}\right)\right)$.

If $\left\{u_{n}\right\}^{*}$ is a singleton, then $\overline{\mathrm{co}}\left\{u_{n}\right\}^{*}=\left\{u_{n}\right\}^{*}$ and $\left\{u^{*}\right\}=\left\{u_{n}\right\}^{*}$; i.e., there is a subsequence of $\left\{u_{n}\right\}$ that converges strongly to $u^{*}$.

Remark. The previous theorem is a type of abstract stability result. These are (usually) obtained with the aid of the Mosco convergence of the sets $K\left(\Phi_{n}\left(u_{n-1}\right)\right)$ to $K\left(\Phi\left(u^{*}\right)\right)$ and the continuity of the operator $A: W \rightarrow W^{\prime}$ (see [41]). Neither of these hypotheses is used to prove the previous theorem.

Copyright $@$ by SIAM. Unauthorized reproduction of this article is prohibited. 
5. Algorithm. Although the method described in this section works for a general operator satisfying the hypotheses of Theorem 4.5, from now on we assume that $A=\Delta_{p}$, as the $p$-Laplacian is related to interesting applications such as (for example) the magnetization of a superconductor (see [31]). Also, we assume that $\Phi: C \subset W \rightarrow L_{\nu}^{\infty}(\Omega)$ is continuous and compact and $C=W$. The algorithmic approach we pursue in order to solve problem $\left(\mathrm{P}_{\mathrm{QVI}}\right)$ consists of solving a sequence of problems with a fixed upper bound, i.e., a sequence of problems $\left(\mathrm{P}_{\mathrm{QVI}}\right)$ with constraint sets $K\left(\varphi_{n}\right)$ for some sequence $\left\{\varphi_{n}\right\} \subset L_{\nu}^{\infty}(\Omega)$.

When $\Phi$ determines a QVI problem (i.e., when $\Phi$ is not constant), then we consider the sequence of solutions $\left\{u_{n}\right\}_{n=0}^{\infty}$ defined as $u_{n}:=S\left(\Delta_{p}, f, \Phi\left(u_{n-1}\right)\right)$ and $u_{0} \in W$ given. Thus, $u_{n}$ solves

$$
\text { find } u \in K\left(\Phi\left(u_{n-1}\right)\right):\left(\Delta_{p}(u)-f, v-u\right) \geq 0 \quad \forall v \in K\left(\Phi\left(u_{n-1}\right)\right) \text {. }
$$

Note that the problem in (5.1) is the first order necessary and sufficient condition of the convex minimization problem

$$
\left.\begin{array}{l}
\min \frac{1}{p}\left(\Delta_{p}(u), u\right)-(f, u) \text { over } u \in W \\
\text { subject to } u \in K\left(\Phi\left(u_{n-1}\right)\right) .
\end{array}\right\}
$$

We propose Algorithm 1 for the sequential minimization of the QVI under consideration. The stopping criteria for our algorithm could be related to the inequality obtained in (4.4), i.e., $\left|u_{n+1}-u_{n}\right|_{W} \leq C\left(\left|\Phi\left(u_{n}\right)-\Phi\left(u_{n-1}\right)\right|_{L^{\infty}(\Omega)}\right)^{1 / p}$ for some $C>0$. This indicates some type of sublinear convergence. Interestingly, in our tests it turned out that we have linear convergence, i.e., $\varlimsup_{n \rightarrow \infty}\left|u_{n+1}-u_{n}\right|_{W} /\left|u_{n}-u_{n-1}\right|_{W}<1$.

For the resolution of step 2 of Algorithm 1 (given in Algorithm 2) consider $J_{\gamma}(u, v):=J(u)+\gamma J^{\mathrm{P}}(u, v)$ with

$$
J(u):=\frac{1}{p} \int_{\Omega}|\nabla u|^{p} \mathrm{~d} \mathbf{x}-(f, u) \text { and } J^{\mathrm{P}}(u, v):=\frac{1}{2} \int_{\Omega}\left|(|\nabla u|-\Phi(v))^{+}\right|^{2} \mathrm{~d} \mathbf{x} .
$$
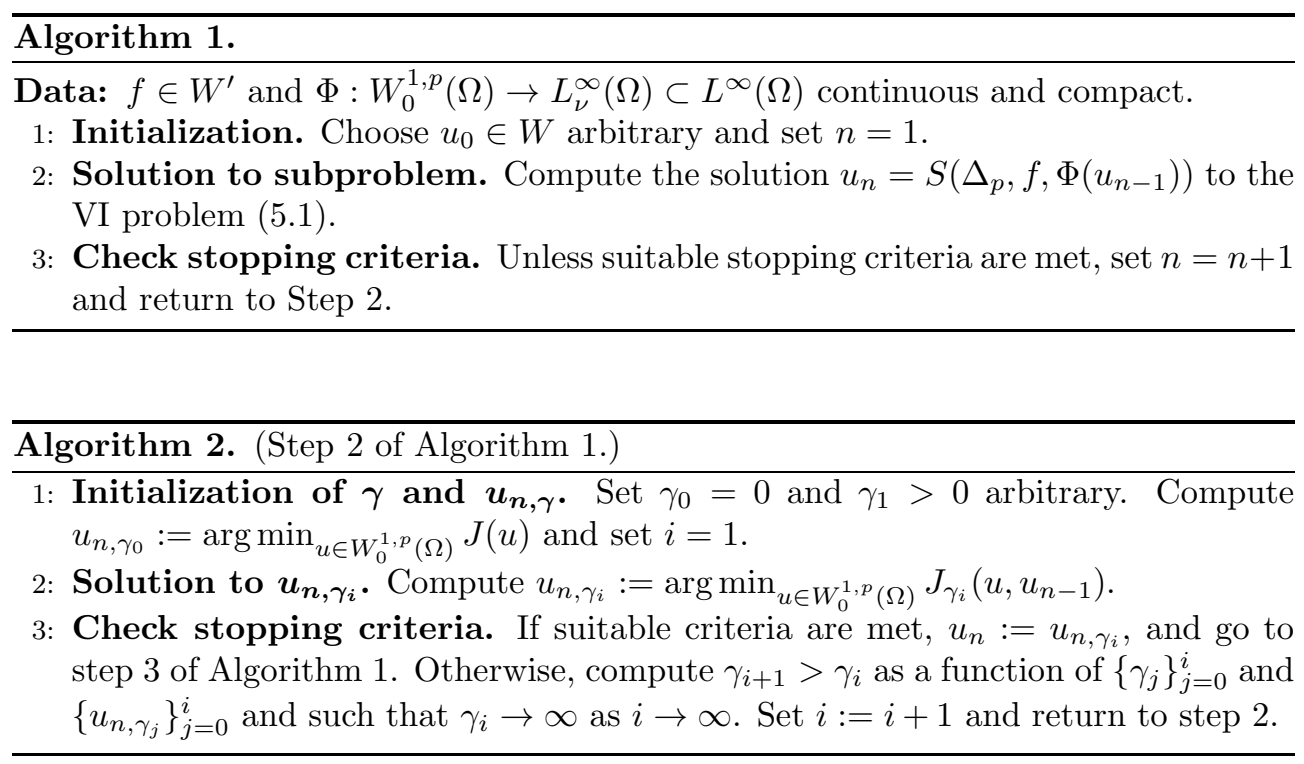
There are several ways of computing $\gamma_{i+1}$ in step 3 of Algorithm 2. The simplest is to consider just $\gamma_{i+1}=\xi \gamma_{i}$ for $i \in \mathbb{N}, \xi>1, \gamma_{0}=0$, and $\gamma_{1}>0$. We consider an adaptive method to perform this iteration in section 6 since Algorithm 2 is highly sensitive with respect to $\gamma$. However, the only requirement for $\left\{\gamma_{i}\right\}$ is $\gamma_{i} \rightarrow \infty$ as $i \rightarrow \infty$.

6. Numerics. We consider the approximation of the problem $\left(\mathrm{P}_{\mathrm{QVI}}\right)$ associated with the $p$-Laplace operator and always use $\Omega:=(0,1) \times(0,1)$ and $p=3$.

Our finite difference approximation scheme has $M^{2}$ uniformly distributed nodes implying the mesh size $h=1 /(M+1)$. At a node $\mathbf{x}_{i j}=\left(x_{i}, x_{j}\right)$, with $x_{i}=i h$ and $x_{j}=j h$ for $1 \leq i, j \leq M$, we approximate $w\left(\mathbf{x}_{i j}\right)$ for $w \in W$ by $w_{i j}=w\left(x_{i}, x_{j}\right)$ and denote by $w^{h}$ the corresponding discrete approximation of $w$ on the given mesh. We approximate the $W$-norm by $\left|w^{h}\right|_{W}^{p}:=\sum_{i, j=1}^{M}\left|\left(D_{-} w^{h}\right)\right|_{i j}^{p} h^{2}$ with $\left(D_{-} w^{h}\right)_{i j}=$ $\frac{1}{h}\left(w_{i j}-w_{(i-1) j}, w_{i j}-w_{i(j-1)}\right)^{\mathrm{T}}$ and $\left|\left(u^{h}, v^{h}\right)^{\mathrm{T}}\right|_{i j}^{2}=u_{i j}^{2}+v_{i j}^{2}$. The discretization of the operator $\Delta_{p}$ and the optimality conditions $\left(\mathrm{OS}_{\gamma}\right)$ are discussed at the end of this section. Also, whenever the pointwise evaluation of $f$ in $\Omega$ is well defined, $\left(f^{h}, w^{h}\right):=\sum_{i, j=1}^{M} f_{i j} w_{i j} h^{2}$. This is the case when $f \in W$, for instance.

In our numerical tests, several choices of $f \in W^{\prime}$ and $\Phi: W \rightarrow L_{\nu}^{\infty}(\Omega)$ are used. In our examples $f \in W \equiv W_{0}^{1, p}(\Omega)$, and we initialize $u_{0}^{h}=f^{h}$ in Algorithm 1. In the case where $f$ is less regular, a different initialization must be used.

Before we analyze Algorithm 2, we describe the termination condition (step 3) of Algorithm 1 in the case when $\Phi$ is not a constant operator.

6.1. The convergence of Algorithm 1. Although there is no proof that Algorithm 1 has linear convergence, we observe in our test runs that the sequence $\left\{\mu_{n}^{h}\right\}_{n=1}^{\infty}$ defined as

$$
\mu_{n}^{h}:=\frac{\left|u_{n+1}^{h}-u_{n}^{h}\right|_{W}}{\left|u_{n}^{h}-u_{n-1}^{h}\right|_{W}} \quad \text { satisfies } \quad \lim _{n \rightarrow \infty} \mu_{n}^{h}=: \mu^{h}<1 .
$$

This implies that $\left\{u_{n}^{h}\right\}_{n=1}^{\infty}$ is a Cauchy sequence with a limit $\bar{u}^{h}$ and that

$$
\left|\bar{u}^{h}-u_{n}^{h}\right|_{W} \leq\left(T\left(\left\{\mu_{n}^{h}\right\}_{n=n+1}^{\infty}\right) \prod_{j=1}^{n} \mu_{j}^{h}\right)\left|u_{1}^{h}-u_{0}^{h}\right|_{W},
$$

with $T\left(\left\{\mu_{n}^{h}\right\}_{n=n+1}^{\infty}\right):=1+\sum_{k=2}^{\infty} \prod_{j=n+1}^{n-1+k} \mu_{j}^{h}$. If there is $\delta \in(0,1)$ such $\mu_{n}^{h}<\delta$ for all $n \in \mathbb{N}$, then $T\left(\left\{\mu_{n}^{h}\right\}_{n=1}^{\infty}\right) \prod_{j=1}^{n} \mu_{j}^{h} \leq \delta^{n} /(1-\delta)$. Since $T\left(\left\{\mu_{n}^{h}\right\}_{n=n+1}^{\infty}\right)=1+\mu_{n+1}^{h}+$ $\mu_{n+1}^{h} \mu_{n+2}^{h}+\cdots$ and $\lim \mu_{n}^{h}=\mu^{h}<1$, then $T\left(\left\{\mu_{n}^{h}\right\}_{n=n+1}^{\infty}\right) \leq 1 /\left(1-\mu_{n+1}^{h}\right) \simeq 1 /\left(1-\mu_{n}^{h}\right)$ for a sufficiently large $n$. Therefore, in our numerics we declare that Algorithm 1 has converged if, for some $n>n_{0}$,

$\left(\mathrm{AL} 1_{\text {convergence }}\right)$

$$
\left.\begin{array}{l}
\max _{n-n_{0} \leq r, s \leq n}\left|\mu_{r}^{h}-\mu_{s}^{h}\right|<\epsilon_{1} \\
\frac{\left|u_{1}^{h}-u_{0}^{h}\right|_{W}}{1-\mu_{n}^{h}} \prod_{j=1}^{n} \mu_{j}^{h}<\epsilon_{2}
\end{array}\right\}
$$

for some prescribed $n_{0} \in \mathbb{N}, \epsilon_{1}>0$, and $\epsilon_{2}>0$; in this case, Algorithm 1 is stopped. In our numerical tests for Examples 3 and 4 , using $n_{0}=4, \epsilon_{1}=1 \mathrm{e}-3$, and $\epsilon_{2}=1 \mathrm{e}-4$, the conditions (AL1 $1_{\text {convergence }}$ ) are satisfied at $n=13$ and $n=11$, respectively. 
6.2. Algorithm 2. The sequence $\left\{\gamma_{m}\right\}_{m=0}^{\infty}$ in Algorithm 2 is defined as follows. First, we bound the minimum and maximum " $\gamma$-steps" by $0<$ MinGammaStep $<$ MaxGammaStep, respectively. We define $\gamma_{0}=0$ and $\gamma_{1}=$ MinGammaStep and assume $\gamma_{m-1}, u_{n, \gamma_{m-1}}^{h}$, and $u_{n-1}^{h}$ are given. Let $\Delta\left(\gamma, \gamma_{m-1}\right):=\gamma-\gamma_{m-1}$, and set

$I\left(\gamma, \gamma_{m-1}, u_{n, \gamma_{m-1}}^{h}, u_{n-1}^{h}\right):=\left(\gamma-\gamma_{m-1}\right) J^{\mathrm{P}}\left(u_{n, \gamma_{m-1}}^{h}, u_{n-1}^{h}\right)-\gamma_{t o l}\left|J_{\gamma_{m}}\left(u_{n, \gamma_{m-1}}^{h}, u_{n-1}^{h}\right)\right|$

(note that $J_{\gamma}(\cdot, \cdot)$ and $J^{\mathrm{P}}(\cdot, \cdot)$ are defined in section 5 ), where $\gamma_{t o l} \in(0,1)$ is a constant which is usually chosen close to 0 , if the map $\Omega \ni \mathbf{x} \mapsto \Phi\left(u_{n-1}^{h}\right)(\mathbf{x})$ is not constant, and close to 1 in the other case. Define the set

$$
\begin{aligned}
\Theta\left(\gamma_{m-1}, u_{n, \gamma_{m-1}}^{h}, u_{n-1}^{h}\right):=\left\{\gamma \in \mathbb{R}^{+}: I\left(\gamma, \gamma_{m-1}, u_{n, \gamma_{m-1}}^{h}, u_{n-1}^{h}\right) \leq 0\right. \text { and } \\
\\
\text { MinGammaStep } \left.\leq \Delta\left(\gamma, \gamma_{m-1}\right) \leq \text { MaxGammaStep }\right\} .
\end{aligned}
$$

For $m \geq 2, \gamma_{m}$ is chosen such that

$$
\left(\Gamma_{\text {update }}\right) \quad \gamma_{m}=\max \Theta\left(\gamma_{m-1}, u_{n, \gamma_{m-1}}^{h}, u_{n-1}^{h}\right) .
$$

After $\gamma_{m}$ is computed according to $\left(\Gamma_{\text {update }}\right), u_{n, \gamma_{m}}^{h}$ is determined as described later in subsection 6.2.1.

Let us further motivate $\left(\Gamma_{\text {update }}\right)$. The condition $I\left(\gamma_{m}, \gamma_{m-1}, u_{n, \gamma_{m-1}}^{h}, u_{n-1}^{h}\right) \leq 0$ is justified as follows. Suppose $u_{n, \gamma} \in W_{0}^{1, p}(\Omega)$ minimizes $J\left(u_{n, \gamma}\right)+\gamma J^{\mathrm{P}}\left(u_{n, \gamma}, u_{n-1}\right)$, and let $V: \mathbb{R}^{+} \rightarrow \mathbb{R}^{+}$be defined as

$$
V(\gamma):=J\left(u_{n, \gamma}\right)+\gamma J^{\mathrm{P}}\left(u_{n, \gamma}, u_{n-1}\right) .
$$

When $p=2$, its Fréchet derivative is $\frac{\mathrm{d} V}{\mathrm{~d} \gamma}(\gamma)=J^{\mathrm{P}}\left(u_{n, \gamma}, u_{n-1}\right)$ (see [24]). In this case, $J^{\mathrm{P}}\left(u_{n, \gamma}^{h}, u_{n-1}^{h}\right)$ is an indicator of how fast the functional is growing with respect to $\gamma$ (and hence $I\left(\gamma, \gamma_{m-1}, u_{n, \gamma_{m-1}}^{h}, u_{n-1}^{h}\right)$ controls the distance, for $\gamma \geq \gamma_{m-1}$, between $V(\gamma)$ and its tangent at the point $\left.\gamma_{m-1}\right)$. In the case of $p>2$, this quantity still seems to be a reliable indicator of the growth behavior of $V$. Note, however, that it is not clear whether $J^{\mathrm{P}}\left(u_{n, \gamma}, u_{n-1}\right)$ is the Fréchet derivative of $V$ when $p \neq 2$.

Let $\mu_{n, m}^{h}:=\left|u_{n, \gamma_{m+1}}^{h}-u_{n, \gamma_{m}}^{h}\right|_{W} /\left|u_{n, \gamma_{m}}^{h}-u_{n, \gamma_{m-1}}^{h}\right|_{W}$. For the meshes studied, i.e., $h=1 / 32,1 / 64,1 / 128,1 / 256$ (and the choice of ( $\Gamma_{\text {update }}$ ) parameters for our numerical runs), we observe that $\lim _{\sup _{m \rightarrow \infty}} \mu_{n, m}^{h}<1$. Based on this, we declare that Algorithm 2 has converged if for some $m \in \mathbb{N}$ the following holds for $\gamma_{m} \geq \gamma_{\min }$ :

$\left(\Gamma_{\text {convergence }}\right)$

$$
\left.\begin{array}{l}
\gamma_{m} J^{\mathrm{P}}\left(u_{n, \gamma_{m}}^{h}, u_{n-1}^{h}\right)<\epsilon_{3}, \\
\left|u_{n, \gamma_{m}}^{h}-u_{n, \gamma_{m-1}}^{h}\right|_{W}<\epsilon_{4}, \\
\mu_{m-1}<\delta_{1}<\mu_{m}
\end{array}\right\}
$$

for some prescribed $\gamma_{\min }>0, \epsilon_{3}>0, \epsilon_{4}>0$, and $0<\delta_{1}<1$; in this case, we stop Algorithm 2 (the inner loop) and return to Algorithm 1 (the outer loop).

The first condition in ( $\left.\Gamma_{\text {convergence }}\right)$ (for a small $\epsilon_{3}>0$ ) guarantees "almost" feasibility of $u_{n, \gamma_{m}}^{h}$ in the $L^{2}(\Omega)$ sense. The second condition (for a small $\epsilon_{4}>0$ ) implies a small variation with respect to the previous iterate, and the third condition (for a $\delta_{1}$ close to 1 ) suggests that the variation in the following iterates will not be significant. The parameter $\gamma_{\min }$ is usually chosen big enough so that the first condition in $\left(\Gamma_{\text {convergence }}\right)$ is not satisfied due only to a small $\gamma$. 
In all the examples that follow we have chosen $\gamma_{\min }=10, \epsilon_{3}=1 \mathrm{e}-5, \epsilon_{4}=1 \mathrm{e}-4$, and $\delta_{1}=0.9$, for which the conditions in $\left(\Gamma_{\text {convergence }}\right)$ are satisfied when $\gamma_{m} \geq$ 500. Also, $m \mapsto \gamma_{m} J^{\mathrm{P}}\left(u_{n, \gamma_{m}}^{h}, u_{n-1}^{h}\right)$ and $m \mapsto\left|u_{n, \gamma_{m}}^{h}-u_{n, \gamma_{m-1}}^{h}\right|_{W}$ are monotonically decreasing, and $\mu_{m}<1$. Hence we consider $u_{n}^{h}:=u_{n \text {,FinalGamma }}^{h}$ with FinalGamma $=$ 1000 .

6.2.1. Step 2 of Algorithm 2. Let $F(u)=\Delta_{p}(u)-f+\gamma \mathfrak{P}(u)$ for $u \in W$, with $\mathfrak{P}(u)=\nabla^{*}\left(|\nabla u|-\Phi\left(u_{n-1}\right)\right)^{+} q(\nabla u) \nabla$ (defined as in Corollary A.3 in Appendix A and where " $\nabla^{*}$ " refers to the adjoint of the operator $\left.\nabla: W_{0}^{1, p}(\Omega) \rightarrow L^{p}(\Omega)^{l}\right)$. If $u^{*}$ satisfies $\left(\mathrm{OS}_{\gamma}\right)$, then $F\left(u^{*}\right)=0$. For the numerical solution of $\left(\mathrm{OS}_{\gamma}\right)$, we next specify the discretization of the system. For this purpose, throughout this section we assume that $u_{n-1}^{h}$ and $\gamma$ are given.

For a (uniform) mesh of size $h$ we define the approximation of the second order elliptic operator $u \mapsto B(a, u):=\nabla^{*}(a(x, y) \nabla u)$ with $a(x, y)>0$ as $[B]^{h}$ given by

$$
\begin{aligned}
-[B]^{h}\left(a^{h}, u^{h}\right)_{i j}=\left(\alpha_{i j}\right. & \left.+\alpha_{(i+1) j}\right)\left(u_{(i+1) j}-u_{i j}\right)-\left(\alpha_{i j}+\alpha_{(i-1) j}\right)\left(u_{i j}-u_{(i-1) j}\right) \\
& +\left(\alpha_{i j}+\alpha_{i(j+1)}\right)\left(u_{i(j+1)}-u_{i j}\right)-\left(\alpha_{i j}+\alpha_{i(j-1)}\right)\left(u_{i j}-u_{i(j-1)}\right),
\end{aligned}
$$

with $\alpha_{i j}=a\left(x_{i}, x_{j}\right) /\left(2 h^{2}\right), 1 \leq i, j \leq M$. This scheme is second order accurate, and the linear map $u^{h} \mapsto B\left(a^{h}, u^{h}\right)$ is symmetric and positive definite (see [27]). Hence, the approximation of $F$, denoted by $[F]^{h}$, is defined as

$$
[F]^{h}\left(u^{h}\right)_{i j}=\left[\Delta_{p}\right]^{h}\left(u^{h}\right)_{i j}-f_{i j}+\gamma[\mathfrak{P}]^{h}\left(u^{h}\right)_{i j},
$$

where

$$
\left[\Delta_{p}\right]^{h}\left(u^{h}\right)_{i j}=[B]^{h}\left(\left|D_{-} u^{h}\right|^{p-2}, u^{h}\right)_{i j},
$$

with $\left(D_{-} u^{h}\right)_{i j}=\frac{1}{h}\left(u_{i j}-u_{(i-1) j}, u_{i j}-u_{i(j-1)}\right)^{\mathrm{T}}$ and $\left|\left(v^{h}, w^{h}\right)\right|_{i j}^{p-2}=\left(v_{i j}^{2}+w_{i j}^{2}\right)^{(p-2) / 2}$, $f_{i j}=f\left(x_{i}, x_{j}\right)$, and

$$
[\mathfrak{P}]^{h}\left(u^{h}\right)_{i j}=[B]^{h}\left(\left(\left|D_{-} u^{h}\right|-[\Phi]^{h}\left(u_{n-1}^{h}\right)\right)^{+} /\left|D_{-} u^{h}\right|, u^{h}\right)_{i j},
$$

with $[\Phi]^{h}\left(v^{h}\right)_{i j}=\Phi\left(v^{h}\right)\left(x_{i}, x_{j}\right),\left(v^{h}\right)_{i j}^{+}=\left(v_{i j}\right)^{+}$, and $\left(u^{h} / w^{h}\right)_{i j}=u_{i j} / w_{i j}$ provided that $w_{i j} \neq 0$.

Assuming that $\left|D_{-} \bar{u}^{h}\right|_{i j} \geq \epsilon>0$ for some prescribed $\epsilon$ and that $\bar{u}^{h}$ solves $[F]^{h}\left(\bar{u}^{h}\right)=0$, following the arguments of the proofs of Theorem A.1 and Corollary A.3 in the discrete setting, one finds that $[F]^{h}$ is semismooth at $\bar{u}^{h}$ (see [40]) and that all $V \in \partial_{\mathrm{B}}[F]^{h}\left(\bar{u}^{h}\right)$ are nonsingular. Here $\partial_{\mathrm{B}} H(x):=\left\{J: J=\lim _{x_{i} \rightarrow x, x_{i} \in D_{H}} \nabla H\left(x_{i}\right)\right\}$, where $H: \mathbb{R}^{m} \rightarrow \mathbb{R}^{n}$ is locally Lipschitz and $D_{H}$ denotes the set where $H$ is differentiable. Therefore, based on this discussion, the Newton iteration

$$
v_{i}=v_{i-1}-V_{i-1}^{-1}[F]^{h}\left(v_{i-1}\right) \text { for } i=1,2, \ldots
$$

with $V_{i-1} \in \partial_{\mathrm{B}}[F]^{h}\left(v_{i-1}\right)$ converges superlinearly to $\bar{u}^{h}$ provided that $v_{0}$ is chosen sufficiently close to $\bar{u}^{h}$ (for a proof of this see, for example, [22] or [40]). Finally, let $u_{n-1}^{h}, u_{n, \gamma_{m-1}}^{h}$, and $\gamma_{m}$ be given. Define $v_{0}=u_{n, \gamma_{m-1}}^{h}$ and perform the iteration

$$
V_{i-1}\left(v_{i}-v_{i-1}\right)=-[F]^{h}\left(v_{i-1}\right) \quad \text { for } i=1,2, \ldots,
$$

where $V_{i-1} \in \partial_{\mathrm{B}}[F]^{h}\left(v_{i-1}\right)$. Then, we define $u_{n, \gamma_{m}}^{h}:=v_{k}$, whenever $\left|v_{k}-v_{k-1}\right|_{W}<$ NewtonTol with NewtonTol $=1 \mathrm{e}-5$. Each iterate $v_{i}$ in (6.1) is computed by the conjugate gradient method with tolerance given by ConjGradTol $=1 \mathrm{e}-3 *$ NewtonTol.

Copyright $@$ by SIAM. Unauthorized reproduction of this article is prohibited. 
6.3. Specification of our test problems and discussion of numerical results. We note that all test problems specified below satisfy the prerequisites of Theorem 3.2. Although Theorem 4.5 (in combination with Theorem B.1) is applicable to a wide range of problems, the $p$-Laplacian in Examples 3 and 4 does not fulfill condition (i) in Theorem B.1 but shows the "contraction" behavior in the numerical tests.

Examples 1 and 2 (VIs). We consider the approximation of two VI-problems on $\Omega \equiv(0.1) \times(0,1)$. The data $f \in W$ and $\Phi: W \rightarrow L_{\nu}^{\infty}(\Omega)$ are given in the following table:

\begin{tabular}{|l|c|c|}
\hline & Example 1 & Example 2 \\
\hline$f\left(x_{1}, x_{2}\right)$ & $\sin \left(2 \pi x_{1}\right) \sin \left(\pi x_{2}\right)$ & $\left(x_{1} x_{2}\left(x_{1}-1\right)\left(x_{2}-1\right)\right)^{2}$ \\
\hline$\Phi(\psi)\left(x_{1}, x_{2}\right)$ & 0.1 & $0.4 \chi_{\Omega_{0}}\left(x_{1}, x_{2}\right)+0.1$ \\
\hline
\end{tabular}

The function $\chi_{\Omega_{0}}$ denotes the characteristic function of the set $\Omega_{0}=(0.5,1) \times$ $(0.5,1)$. In these examples we use the mesh size $h=1 / 256$ for computing the finite difference approximation to the solution of the respective VIs. The computed numerical solutions $\bar{u}^{h}$ are shown in Figures 6.1(e) and 6.1(f) for Examples 1 and 2, respectively. The approximations of the corresponding active sets (i.e., $\mathcal{A}=\{\mathrm{x} \in$ $\Omega:|\nabla \bar{u}(\mathbf{x})|-\Phi(\bar{u})(\mathbf{x})=0\}$, where $\bar{u}$ solves the VI) are shown in Figures 6.1(c) and $6.1(\mathrm{~d})$, respectively.

Next, we study the stability of the Newton iteration with respect to mesh refinements. For this purpose we use a sequence of uniform meshes with $h_{k}=2^{-k}$ with $k=5,6,7,8$. The parameters for $\left(\Gamma_{\text {update }}\right)$, common to both examples, are $\gamma_{t o l}=0.2$ and MinGammaStep=0.01. We use MaxGammaStep=100 and MaxGammaStep=10 for Examples 1 and 2, respectively. The number of Newton iterations (until successful termination for solving step 2 of Algorithm 1) with respect to $\gamma$ in Figures 6.1(a) and 6.1(b) show little variation with respect to $h$. This suggests mesh independence of our semismooth Newton solver in Algorithm 2. Figure 6.1(b) shows the number of Newton iterations only for $0 \leq \gamma \leq 300$. For $\gamma>300$ and for all meshes, only three iterations are required.

Examples 3 and 4 (QVIs). The mesh size in these examples is $h=1 / 256$. The data $f \in W$ and $\Phi: W \rightarrow L_{\nu}^{\infty}(\Omega)$ for Examples 3 and 4 are given as follows:

\begin{tabular}{|l|c|c|}
\hline & Example 3 & Example 4 \\
\hline$f\left(x_{1}, x_{2}\right)$ & $\left(x_{1} x_{2}\left(x_{1}-1\right)\left(x_{2}-1\right)\right)^{2}$ & $\sin \left(2 \pi x_{1}\right) \sin \left(\pi x_{2}\right)$ \\
\hline$\Phi(\psi)\left(x_{1}, x_{2}\right)$ & $2\left|\int_{\Omega} \psi(\mathbf{x}) \mathrm{d} \mathbf{x}\right|+0.1$ & $10 \phi_{1}(\mathbf{x})\left|\int_{\Omega} \phi_{2}(\mathbf{x}) \psi(\mathbf{x}) \mathrm{d} \mathbf{x}\right|+0.05$ \\
\hline
\end{tabular}

The variable " $\mathrm{x}$ " is shorthand notation for " $\left(x_{1}, x_{2}\right)$." The functions $\phi_{1}$ and $\phi_{2}$ are defined by

$$
\phi_{1}\left(x_{1}, x_{2}\right):=e^{-5\left(\left(x_{1}-\frac{1}{3}\right)^{2}+\left(x_{2}-\frac{1}{3}\right)^{2}\right)}, \quad \phi_{2}\left(x_{1}, x_{2}\right):=10 \chi_{\Omega_{0}}\left(x_{1}, x_{2}\right)+0.1,
$$

where $\chi_{\Omega_{0}}$ denotes the characteristic function of the set $\Omega_{0}=(0.5,1) \times(0.5,1)$.

The parameters for $\left(\Gamma_{\text {update }}\right)$ are given by the following:

\begin{tabular}{|l|c|c|}
\hline & Example 3 & Example 4 \\
\hline$\gamma_{\text {tol }}$ & 0.1 & $1 \mathrm{e}-4$ \\
\hline MinGammaStep & 0.1 & 1 \\
\hline MaxGammaStep & 100 & 10 \\
\hline
\end{tabular}

The numerical results are shown in Figures 6.2 and 6.3, respectively.

Our theory does not ensure linear convergence of the fixed-point iteration for approximating the solution of the QVI. However, we observe numerically that the quotient $\mu_{n}:=\left|u_{n+1}^{h}-u_{n}^{h}\right|_{W} /\left|u_{n}^{h}-u_{n-1}^{h}\right|_{W}$ approaches 0.3139 in Example 3 and 0.3055 in Example 4 as observed in Figures 6.2(b) and 6.3(b), respectively. This implies a 


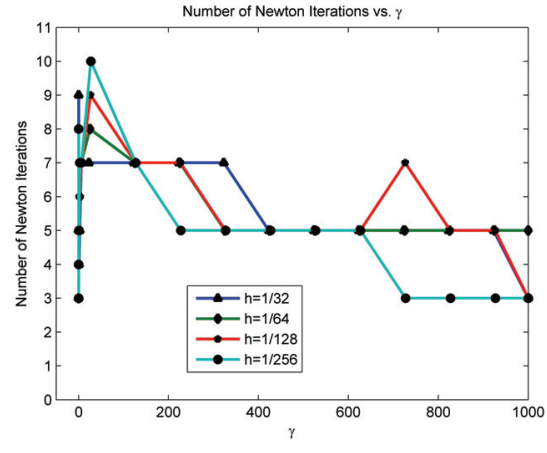

(a) Newton iterations. Example 1.

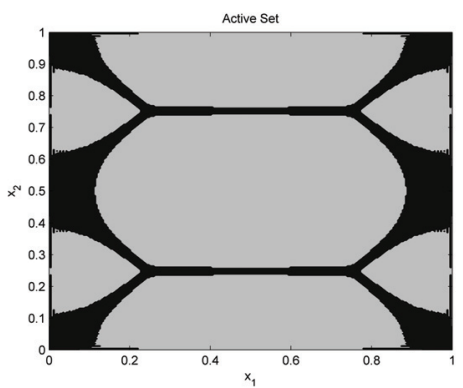

(c) Active set. Example 1.

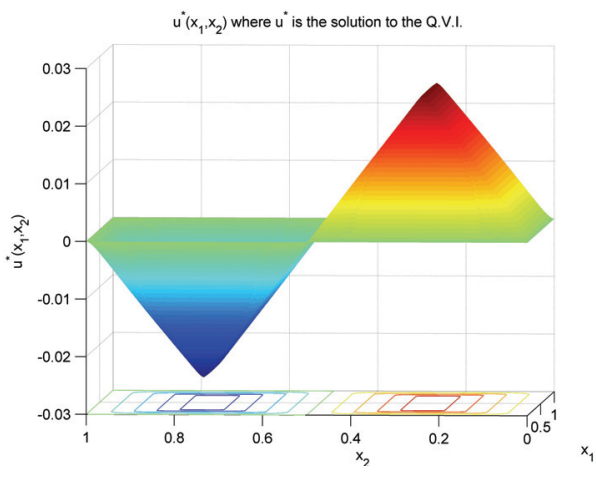

(e) Approximate solution. Example 1.

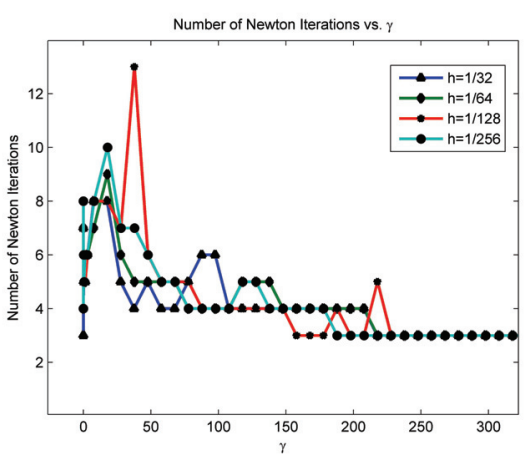

(b) Newton iterations. Example 2.

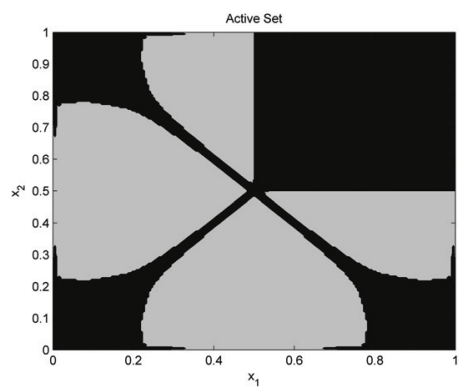

(d) Active set. Example 2 .

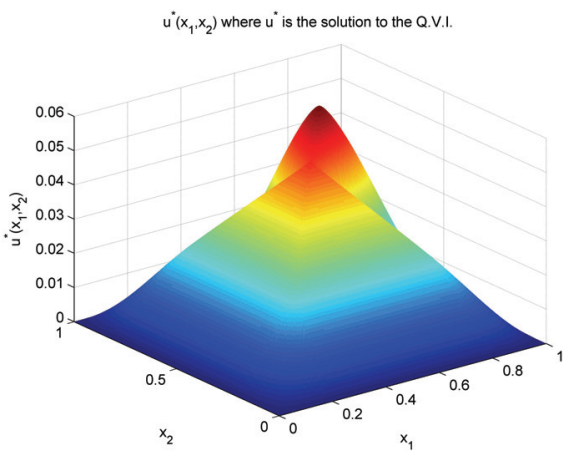

(f) Approximate solution. Example 2.

FIG. 6.1. Number of Newton iterations with respect to $\gamma$ for (a) Example 1 and (b) Example 2. Active set (grey) $\mathcal{A}:=\left\{\mathbf{x} \in \Omega:\left|\nabla u^{*}(\mathbf{x})\right|-\Phi(\mathbf{x})=0\right\}$, where $u^{*}$ is the approximated solution to the VI for (c) Example 1 and (d) Example 2. Approximation to the final solution of the VI for (e) Example 1 and (f) Example 2.

much faster convergence than expected from the previously described relation $\mid u_{n+1}^{h}-$ $\left.u_{n}^{h}\right|_{W} \leq C_{\phi}\left|\Phi\left(u_{n}^{h}\right)-\Phi\left(u_{n-1}^{h}\right)\right|_{L^{\infty}(\Omega)}^{1 / p}$. Indeed, our results appear to indicate $\mid u_{n+1}^{h}-$ $\left.u_{n}^{h}\right|_{W} /\left|\Phi\left(u_{n}^{h}\right)-\Phi\left(u_{n-1}^{h}\right)\right|_{L^{\infty}(\Omega)}^{1 / p} \rightarrow 0$ as $n$ increases in both examples (see Figures $6.2(\mathrm{a})$ and $6.3(\mathrm{~b}))$.

The behavior of $n \mapsto J\left(u_{n}^{h}\right)$, where $n$ is the iteration number and $J(u):=\int|\nabla u|^{p} \mathrm{~d} \mathbf{x} / p-\int f u \mathrm{~d} \mathbf{x}$, is shown in Figures 6.2(c) and 6.3(c) for Examples 3 and 4 , respectively. 


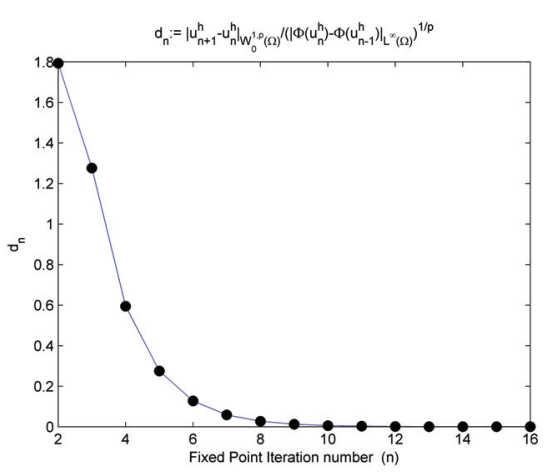

(a)

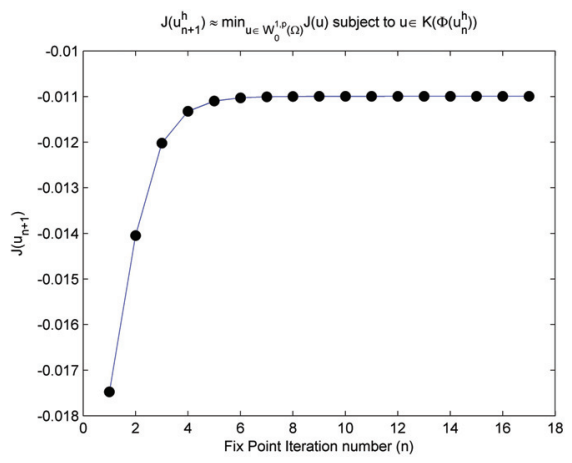

(c)

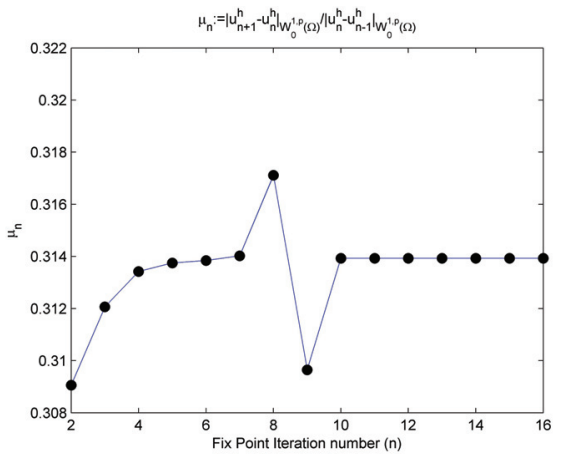

(b)

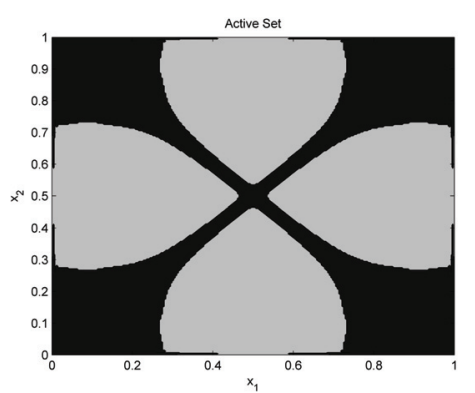

(d)

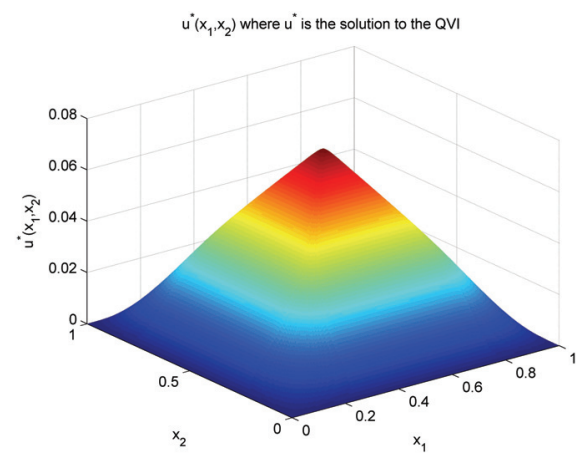

(e)

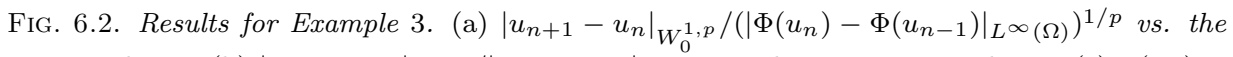
iteration number $n$. (b) $\left|u_{n+1}-u_{n}\right|_{W_{0}^{1, p}} /\left|u_{n}-u_{n-1}\right|_{W_{0}^{1, p}}$ vs. the iteration number $n$. (c) $J\left(u_{n}\right)=$ $\min _{u \in W_{0}^{1, p}} J(u)$ subject to $u \in K\left(\Phi\left(u_{n-1}\right)\right)$ vs. iteration number $n$. (d) Active set (grey) $\mathcal{A}$. (e) Approximate solution to the QVI.

Copyright $@$ by SIAM. Unauthorized reproduction of this article is prohibited. 


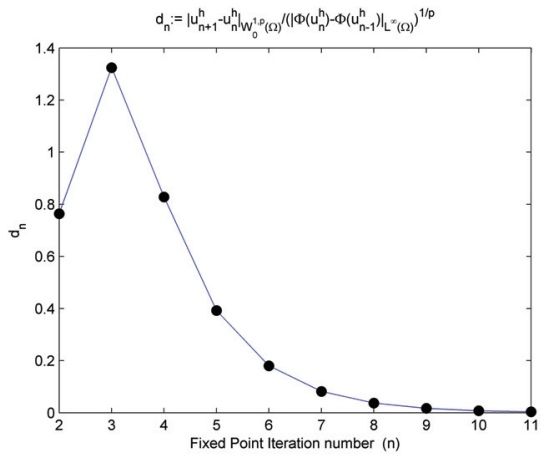

(a)

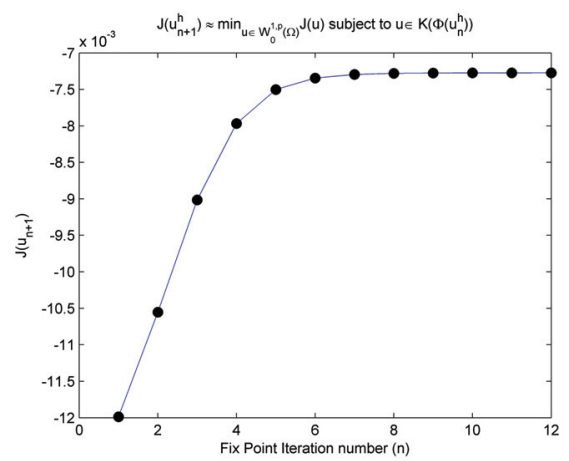

(c)

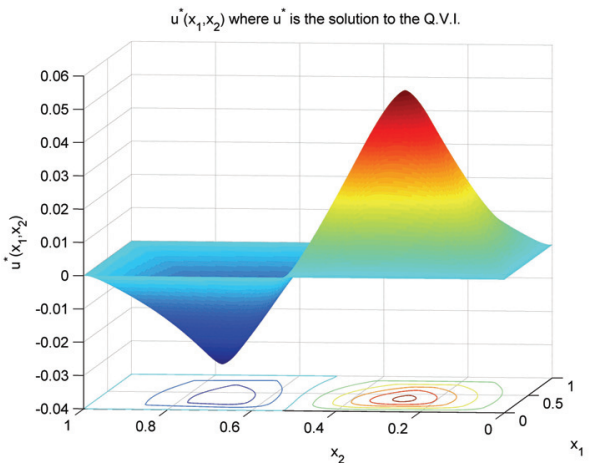

(e)

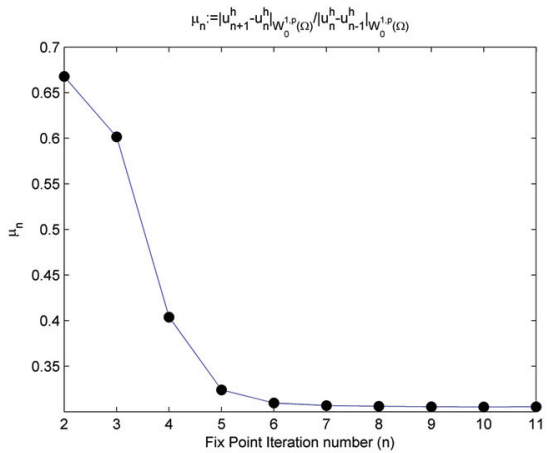

(b)

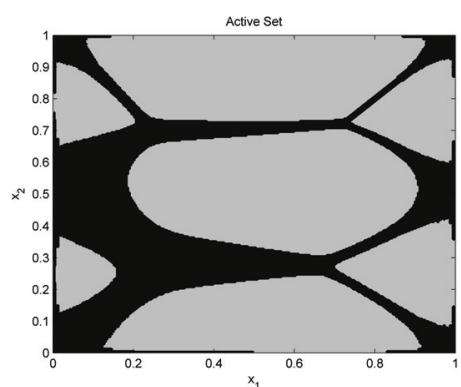

(d)

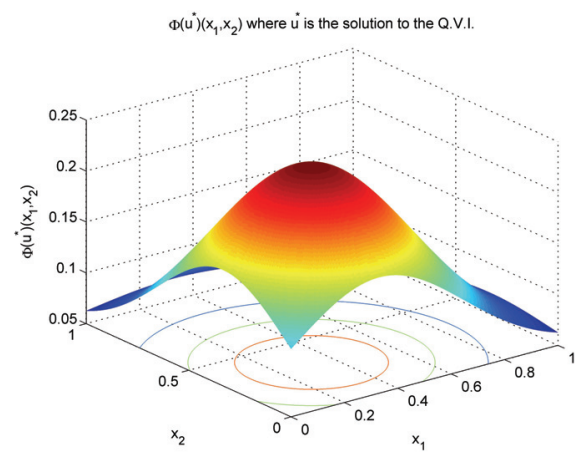

(f)

FIG. 6.3. Results for Example 3. (a) $\left|u_{n+1}-u_{n}\right|_{W_{0}^{1, p}} /\left(\left|\Phi\left(u_{n}\right)-\Phi\left(u_{n-1}\right)\right|_{L^{\infty}(\Omega)}\right)^{1 / p}$ vs. the iteration number $n$. (b) $\left|u_{n+1}-u_{n}\right|_{W_{0}^{1, p}} /\left|u_{n}-u_{n-1}\right|_{W_{0}^{1, p}}$ vs. the iteration number $n$. (c) $J\left(u_{n}\right)=$ $\min _{u \in W_{0}^{1, p}} J(u)$ subject to $u \in K\left(\Phi\left(u_{n-1}\right)\right)$ vs. iteration number $n$. (d) Active set (grey) $\mathcal{A}$. (e) approximate solution to the QVI. (f) $\Phi\left(u^{*}\right)$, where $u^{*}$ is the approximated solution to the $Q V I$.

Copyright $@$ by SIAM. Unauthorized reproduction of this article is prohibited. 
The approximation of the active set $\mathcal{A}=\{\mathbf{x} \in \Omega:|\nabla \bar{u}|-\Phi(\bar{u})=0\}$, where $\bar{u}$ solves the QVI of interest, is shown in Figures 6.2(d) and 6.3(d). Here, $\bar{u}$ is approximated by $u_{n^{*}}^{h}$, which satisfies conditions (AL1 $1_{\text {convergence }}$ ) for some $n^{*} \in \mathbb{N}$. The values of $n^{*}$ are 13 and 11 for Examples 3 and 4, respectively.

7. Discussion and open problems. In section 6 , we provided details on the implementation of the generalized Newton method for solving a nonsmooth equation. This equation was related to the first order optimality system of step 2 of Algorithm 1. Clearly, from a functional analytic and also a numerical analysis (mesh independence) point of view, it might be of interest to study the subproblem solver in the original function space setting. See, e.g., [21, 23, 24, 22] for such an analysis in the context of optimization problems with PDE constraints or path-following techniques for classes of variational inequalities. In our context this analysis is hindered by the following fact. Let $F$ be given by

$$
F(u)=\Delta_{p}(u)-f+\gamma \mathfrak{P}(u),
$$

where $\Delta_{p}$ is the monotone operator defined in $(2.2)$ and $\mathfrak{P}(u)=$ $\nabla^{*}\left(|\nabla u|-\Phi\left(u_{n-1}\right)\right)^{+} q(\nabla u) \nabla$ as in Corollary A.3. Then $F$ is Newton (or slant) differentiable, i.e., semismooth, as a mapping from $W_{0}^{1, p}(\Omega)$ to $\left(W_{0}^{1, s^{\prime}}\right)^{\prime}$ for $3 \leq 3 s \leq p<\infty$, where $1 / s+1 / s^{\prime}=1$ (for a proof, see Theorem A.1 and Corollary A.3) with a Newton (or slant) derivative $G_{F}$ given by

$$
G_{F}(u)=D \Delta_{p}(u)+\gamma G_{\mathfrak{P}}(u),
$$

where $D \Delta_{p}$ is defined as in (A.1) and $G_{\mathfrak{P}}$ as in (A.9). We refer the reader to [22, $26,40]$ for the notion of a Newton (or slant) derivative. The fact that the range space is $\left(W_{0}^{1, s^{\prime}}(\Omega)\right)^{\prime}$ implies a fundamental difficulty associated with the Newtontype iteration, for which we require the Newton derivative of $F$ to have a uniformly bounded inverse in some neighborhood of $u^{*} \in W$ with $F\left(u^{*}\right)=0$. At this point, the latter property is still an open question, and it is not clear whether it is possible to add a "lifting step" to the algorithm (as done in [24]) to overcome this difficulty.

The behavior of $\mu_{n}^{h}=\left|u_{n+1}^{h}-u_{n}^{h}\right|_{W} /\left|u_{n}^{h}-u_{n-1}^{h}\right|_{W}$ is an interesting feature of Examples 3 and 4 . In addition to showing linear convergence, it satisfies $\mu_{n}^{h / 2}-\mu_{n}^{h} \leq 0$ for $h=1 / 32,1 / 64,1 / 128$ (for $n \geq 2$ ), and this difference gets smaller as $h$ decreases. The observed linear convergence suggests that the bound established in Lemma 4.1 is not optimal, i.e., the Lipschitz continuity of the mapping $u \mapsto S(A, f, \Phi(u)$ ) (obtained in Theorem B.1) might hold under weaker conditions on $A$ and $\Phi$, in some neighborhood of a solution to problem $\left(\mathrm{P}_{\mathrm{QVI}}\right)$.

In Examples 1-3 the quantity $\mu_{n, m}^{h}:=\left|u_{n, \gamma_{m+1}}^{h}-u_{n, \gamma_{m}}^{h}\right|_{W} /\left|u_{n, \gamma_{m}}^{h}-u_{n, \gamma_{m-1}}^{h}\right|_{W}$ decreases monotonically and $\mu_{n, m}^{h}<1$ for the region $2 \leq m \lesssim 8$ (and for $n \geq 2$ in Example 3). This suggest that in this region we have superlinear convergence of $m \mapsto u_{n, \gamma_{m}}^{h}$ to $u_{n}^{h}$, and this is reflected in the relatively low values of $\gamma$ for which the conditions in $\left(\Gamma_{\text {convergence }}\right)$ are met. In Example 4 , although $\mu_{n, m}^{h}$ does not decrease, it satisfies $0.1 \leq \mu_{n, m}^{h} \leq 0.5$ for $2 \leq m \lesssim 8$. The behavior described above seems to be independent of the mesh size.

The choice of parameters in $\left(\Gamma_{\text {update }}\right)$ is crucial for the convergence of the algorithm in each of the examples. Our observation is that if $\varphi$ in $K(\varphi)$ is spatially variant in $\Omega$, then the strategy should be conservative (i.e., $\gamma_{t o l}$, MinGammaStep, and MaxGammaStep should be relatively small). For Examples 1 and 3, the algorithm appears much more robust with respect to the parameters in $\left(\Gamma_{\text {update }}\right)$. 
Appendix A. Differentiability properties. We study differentiability properties of $\Delta_{p}: W \rightarrow W^{\prime}$,

$$
\left(\Delta_{p}(u), v\right)=\int_{\Omega}|\nabla u|^{p-2}(\nabla u \cdot \nabla v) \mathrm{d} \mathbf{x},
$$

for all $u, v \in W \equiv W_{0}^{1, p}(\Omega)$ and of $\mathfrak{P}: W \rightarrow\left(W_{0}^{1, s^{\prime}}(\Omega)\right)^{\prime}$ (for some $s^{\prime}$ ),

$$
(\mathfrak{P}(u), w)=\int_{\Omega^{+}(u)}(|\nabla u|-\varphi)^{+} \frac{(\nabla u \cdot \nabla w)}{|\nabla u|} \mathrm{d} \mathbf{x},
$$

for all $u \in W$ and $w \in W_{0}^{1, s^{\prime}}(\Omega)$, where $\varphi \in L_{\nu}^{\infty}(\Omega):=\left\{v \in L^{\infty}(\Omega):|v(\mathbf{x})| \geq \nu>\right.$ 0 a.e. for $\mathbf{x} \in \Omega\}$ and $\Omega^{+}(u):=\{\mathbf{x} \in \Omega:|\nabla u(\mathbf{x})|>0\}$.

\section{A.1. Fréchet Differentiability of the $p$-Laplacian.}

Theorem A.1. The map $\Delta_{p}: W \rightarrow W^{\prime}$ is continuously Fréchet differentiable for $2 \leq p<\infty$ with derivative $D \Delta_{p}(u) \in \mathscr{L}\left(W, W^{\prime}\right)$ at $u \in W$, given by

$$
\left(D \Delta_{p}(u) h, z\right)=\int_{\Omega^{+}(u)}(p-2)|\nabla u|^{p-4}(\nabla u \cdot \nabla h)(\nabla u \cdot \nabla z)+|\nabla u|^{p-2}(\nabla h \cdot \nabla z) \mathrm{d} \mathbf{x}
$$

for all $h, z \in W$ with $\Omega^{+}(u)=\{\mathbf{x} \in \Omega:|\nabla u(\mathbf{x})|>0\}$.

Proof. Suppose $p>2$ (when $p=2$ the result is standard).

Step 1: $D \Delta_{p}(u) h$ is the strong Gâteaux derivative at $u \in W$ in direction $h \in W$. For fixed $\mathbf{x} \in \Omega$, the map $t \mapsto|\nabla u(\mathbf{x})+t \nabla h(\mathbf{x})|^{p-2}(\nabla u(\mathbf{x})+t \nabla h(\mathbf{x}))^{\mathrm{T}}$ defines a differentiable function $G(\cdot, \mathbf{x}): \mathbb{R} \rightarrow \mathbb{R}^{l}$ as

$$
G(t, \mathbf{x})=|\nabla u(\mathbf{x})+t \nabla h(\mathbf{x})|^{p-2}(\nabla u(\mathbf{x})+t \nabla h(\mathbf{x}))^{\mathrm{T}} .
$$

Suppressing "( $\mathbf{x})$ " for the sake of brevity, its derivative with respect to " $t$ " is given by

$$
\begin{gathered}
G^{\prime}(t, \cdot)=(p-2)|\nabla u+t \nabla h|^{p-4}\left((\nabla u+t \nabla h)^{\mathrm{T}} \nabla h\right)(\nabla u+t \nabla h)^{\mathrm{T}} \\
+|\nabla u+t \nabla h|^{p-2}(\nabla h)^{\mathrm{T}}
\end{gathered}
$$

when $\nabla u(\mathbf{x})+t \nabla h(\mathbf{x}) \neq 0$ and $G^{\prime}(t, \mathbf{x})=0$ otherwise. If $\mathbf{x} \notin \Omega^{+}(u)$, then $G^{\prime}(0, \mathbf{x})=0$.

Suppose that $0<|t|<1$. Then the mean value theorem implies that

$$
|G(t, \mathbf{x})-G(0, \mathbf{x})| \leq(p-1)(|\nabla u(\mathbf{x})|+|\nabla h(\mathbf{x})|)^{p-2}|\nabla h(\mathbf{x})||t| .
$$

Also, for $0<|t|<1$, we have

$$
\begin{array}{r}
\left|\left(\frac{\Delta_{p}(u+t h)-\Delta_{p}(u)}{t}-D \Delta_{p}(u) h, z\right)\right| \leq q \int_{\Omega}\left|\frac{G(t, \mathbf{x})-G(0, \mathbf{x})}{t}-G^{\prime}(0, \mathbf{x})\right||\nabla z| \mathrm{d} \mathbf{x} \\
\leq\left(\int_{\Omega}\left|\frac{G(t, \mathbf{x})-G(0, \mathbf{x})}{t}-G^{\prime}(0, \mathbf{x})\right|^{\frac{p}{p-1}} \mathrm{~d} \mathbf{x}\right)^{\frac{p-1}{p}}|z|_{W} .
\end{array}
$$

Since $(|\nabla u|+|\nabla h|)^{p-2}|\nabla h| \in L^{q}(\Omega)$ and $|\nabla u|^{p-2}|\nabla h| \in L^{q}(\Omega)$ with $\frac{1}{q}+\frac{1}{p}=1$ so that $q=\frac{p}{p-1}$, then

$$
\begin{aligned}
& \left|\frac{G(t, \mathbf{x})-G(0, \mathbf{x})}{t}-G^{\prime}(0, \mathbf{x})\right|^{\frac{p}{p-1}} \\
& \quad \leq\left((p-1)(|\nabla u|+|\nabla h|)^{p-2}|\nabla h|+(p-1)|\nabla u|^{p-2}|\nabla h|\right)^{\frac{p}{p-1}} \in L^{1}(\Omega) .
\end{aligned}
$$

Copyright $@$ by SIAM. Unauthorized reproduction of this article is prohibited. 
Applying Lebesgue's bounded convergence theorem, we observe that $D \Delta_{p}(u) h$ is the strong Gâteaux differential of $\Delta_{p}$ at $u$ in the direction $h$, i.e.,

$$
\lim _{t \rightarrow 0} \frac{\Delta_{p}(u+t h)-\Delta_{p}(u)}{t}=D \Delta_{p}(u) h .
$$

Step 2: Existence and continuity of the Fréchet derivative. We will show that $u \mapsto$ $D \Delta_{p}(u)$ is continuous, and thus $D \Delta_{p}(u)$ is the Fréchet derivative of $\Delta_{p}: W \rightarrow W^{\prime}$ at $u \in W$. It is convenient to write the pairing $\left(D \Delta_{p}(u) h, z\right)$ with the use of transposes as

$$
\left(D \Delta_{p}(u) h, z\right)=\int_{\Omega}\left((p-2)\left(\nabla h^{\mathrm{T}} \nabla u\right)|\nabla u|^{p-4} \chi_{\Omega^{+}(u)} \nabla u^{\mathrm{T}}+|\nabla u|^{p-2} \nabla h^{\mathrm{T}}\right) \nabla z \mathrm{~d} \mathbf{x},
$$

where $\chi_{\Omega^{+}(u)}$ is the characteristic function of the set $\Omega^{+}(u)$. Consider $k: \mathbb{R}^{l} \rightarrow \mathbb{R}$ and $k_{i, j}: \mathbb{R}^{l} \rightarrow \mathbb{R}$, for $1 \leq i, j \leq l$, defined as

$$
k(\mathbf{x})=|\mathbf{x}|^{p-2} \quad \text { and } \quad k_{i, j}(\mathbf{x})= \begin{cases}|\mathbf{x}|^{p-4}\left(\mathbf{x} \cdot e_{i}\right)\left(\mathbf{x} \cdot e_{j}\right), & \mathbf{x} \neq 0 \\ 0, & \mathbf{x}=0\end{cases}
$$

where $e_{k}$ is the vector with a one in the $k$ th position and zero otherwise. All functions $k$ and $k_{i, j}$, for $1 \leq i, j \leq l$, are continuous and satisfy

$$
\max \left(\left|k_{i, j}(\mathbf{x})\right|,|k(\mathbf{x})|\right) \leq|\mathbf{x}|^{p-2}=|\mathbf{x}|^{\frac{p}{q}} \quad \text { for } 1 \leq i, j \leq l
$$

with $q=\frac{p}{p-2}$. Hence all $k_{i, j}$ and $k$ are $(p, q)$-Carathéodory (see [38]). Therefore, the Nemytskii (or superposition) operators defined by $N_{i, j}(w)(\mathbf{x}):=k_{i, j}(w(\mathbf{x}))$ and $N(w)(\mathbf{x}):=k(w(\mathbf{x}))$ with $N_{i, j}: L^{p}(\Omega)^{l} \rightarrow L^{\frac{p}{p-2}}(\Omega)$ for $1 \leq i, j \leq l$ and $N: L^{p}(\Omega)^{l} \rightarrow$ $L^{\frac{p}{p-2}}(\Omega)$ are well defined and continuous (see, for example, [38] or [5]).

We observe, due to the equivalency of norms in finite dimensions, that

$$
\begin{aligned}
& \left|\left(D \Delta_{p}(u) h-D \Delta_{p}(v) h, z\right)\right| \\
& \leq\left.\int_{\Omega}(p-2)|\nabla u| \nabla u\right|^{p-4} \chi_{\Omega^{+}(u)} \nabla u^{\mathrm{T}}-\nabla v|\nabla v|^{p-4} \chi_{\Omega^{+}(v)} \nabla v^{\mathrm{T}}|| \nabla h|| \nabla z \mid \\
& \quad+\left.|| \nabla u\right|^{p-2}-|\nabla v|^{p-2}|| \nabla h|| \nabla z \mid \mathrm{d} \mathbf{x} \\
& \leq\left. C(p-2) \sum_{i, j=1}^{l} \int_{\Omega}|| \nabla u\right|^{p-4} \chi_{\Omega^{+}(u)}\left(\nabla_{i} u\right)\left(\nabla_{j} u\right)-|\nabla v|^{p-4} \chi_{\Omega^{+}(v)}\left(\nabla_{i} v\right)\left(\nabla_{j} v\right)|| \nabla h|| \nabla z \mid \\
& \quad+\left.|| \nabla u\right|^{p-2}-|\nabla v|^{p-2}|| \nabla h|| \nabla z \mid \mathrm{d} \mathbf{x}
\end{aligned}
$$

for some $C>0$ and where $\nabla_{i} u=\nabla u \cdot e_{i}$ for $1 \leq i \leq l$. Hence, using Hölder's 
inequality, we obtain

$$
\begin{aligned}
& \left|\left(D \Delta_{p}(u) h-D \Delta_{p}(v) h, z\right)\right| \\
& \leq C\left(( p - 2 ) \sum _ { i , j = 1 } ^ { l } \left(\int_{\Omega}|\nabla u|^{p-4} \chi_{\Omega^{+}(u)}\left(\nabla_{i} u\right)\left(\nabla_{j} u\right)\right.\right. \\
& \left.-\left.|\nabla v|^{p-4} \chi_{\Omega^{+}(v)}\left(\nabla_{i} v\right)\left(\nabla_{j} v\right)\right|^{\frac{p}{p-2}} \mathrm{~d} \mathbf{x}\right)^{\frac{p-2}{p}} \\
& \left.+\left(\left.\int_{\Omega}|| \nabla u\right|^{p-2}-\left.|\nabla v|^{p-2}\right|^{\frac{p}{p-2}} \mathrm{~d} \mathbf{x}\right)^{\frac{p-2}{p}}\right)|h|_{W}|z|_{W} .
\end{aligned}
$$

As a consequence, we infer

$$
\begin{aligned}
& \left|D \Delta_{p}(u)-D \Delta_{p}(v)\right|_{\mathscr{L}\left(W, W^{\prime}\right)} \\
& \leq C\left((p-2) \sum_{i, j=1}^{l}\left|N_{i, j}(\nabla u)-N_{i, j}(\nabla v)\right|_{L^{q}(\Omega)}+|N(\nabla u)-N(\nabla v)|_{L^{q}(\Omega)}\right),
\end{aligned}
$$

with $q=\frac{p}{p-2}$. Suppose now that $u_{n} \rightarrow u$ in $W$. This implies that $\nabla u_{n} \rightarrow \nabla u$ in $L^{p}(\Omega)^{l}$, and since each one of the mappings $N_{i, j}: L^{p}(\Omega)^{l} \rightarrow L^{\frac{p}{p-2}}(\Omega)$ for $1 \leq$ $i, j \leq l$ and $N: L^{p}(\Omega)^{l} \rightarrow L^{\frac{p}{p-2}}(\Omega)$ is continuous, the above inequality implies that $D \Delta_{p}\left(u_{n}\right) \rightarrow D \Delta_{p}(u)$. Therefore $D \Delta_{p}(\cdot): W \rightarrow \mathscr{L}\left(W, W^{\prime}\right)$ is continuous, and hence $D \Delta_{p}(u)$ is the Fréchet derivative of the map $u \mapsto \Delta_{p}(u)$ at $u$.

Newton derivative of the penalty term. Consider $P(u):=b(u) q(u)$ with $b(u)(\mathbf{x})=(|u(\mathbf{x})|-\varphi(\mathbf{x}))^{+}$, where $\varphi(\mathbf{x}) \geq \nu>0$ and $q(u)(\mathbf{x})=\frac{u(\mathbf{x})}{|u(\mathbf{x})|}$ when $u(\mathbf{x}) \neq 0$. Suppose $\Omega \subset \mathbb{R}^{l}$ is an open and bounded domain. We know that $b: L^{p}(\Omega)^{l} \rightarrow L^{s}(\Omega)$ for $1 \leq s<p \leq \infty$, with a Newton derivative (see, for example, [26] for a definition) $G_{b}(u) \in \mathscr{L}\left(L^{p}(\Omega)^{l}, L^{s}(\Omega)\right)$ given by

$$
G_{b}(u)(\mathbf{x})=G_{\max }(|u(\mathbf{x})|-\varphi) \frac{u^{\mathrm{T}}(\mathbf{x})}{|u(\mathbf{x})|}
$$

when $u(\mathbf{x}) \neq 0$ and where $G_{\max }$ is the Newton derivative of $\max (\cdot, 0): L^{p}(\Omega)^{l} \rightarrow$ $L^{s}(\Omega)$ (see [24] and [22]). Define $Q(u)$ as

$$
Q(u)(\mathbf{x})=\frac{1}{|u(\mathbf{x})|}\left(\operatorname{id}-\frac{u(\mathbf{x}) u^{\mathrm{T}}(\mathbf{x})}{|u(\mathbf{x})|^{2}}\right)
$$

when $u(\mathbf{x}) \neq 0$ and where id is the identity mapping. We have the following result.

TheOrem A.2. Let $p$ and $s$ satisfy $3 \leq 3 s \leq p<\infty$; then $G_{P}(u): L^{p}(\Omega)^{l} \rightarrow$ $L^{s}(\Omega)^{l}$, given by

$$
G_{P}(u)=q(u) G_{b}(u)+b(u) Q(u),
$$

is a Newton derivative of $P$.

Copyright $@$ by SIAM. Unauthorized reproduction of this article is prohibited. 
Proof. Consider

$$
\begin{aligned}
P(u+h)-P(u)-G_{P}(u+h) h= & b(u+h)(q(u+h)-q(u)-Q(u+h) h) \\
& +(q(u)-q(u+h))(b(u+h)-b(u)) \\
& +q(u+h)\left(b(u+h)-b(u)-G_{b}(u+h) h\right) \\
= & I+I I+I I I .
\end{aligned}
$$

We start by estimating $I$. Define

$$
\Omega_{\nu}(h)=\{\mathbf{x} \in \Omega:|(u+h)(\mathbf{x})|>\nu\} .
$$

We observe that if $\mathbf{x} \in \Omega \backslash \Omega_{\nu}(h)$, then $b(u+h)(\mathbf{x})=0$. Hence $|I|_{L^{s}(\Omega)^{l}}=|I|_{L^{s}\left(\Omega_{\nu}(h)\right)^{l}}$. We partition $\Omega_{\nu}(h)=\Omega_{\nu}^{+}(h) \cup \Omega_{\nu}^{-}(h)$ with

$$
\Omega_{\nu}^{+}(h):=\left\{\mathbf{x} \in \Omega_{\nu}(h):|u(\mathbf{x})|>\frac{\nu}{2}\right\} \text { and } \Omega_{\nu}^{-}(h):=\Omega_{\nu}(h) \backslash \Omega_{\nu}^{+}(h) .
$$

If $\mathbf{x} \in \Omega_{\nu}^{-}(h)$, then $|u(\mathbf{x})| \leq \nu / 2$ and $|(u+h)(\mathbf{x})|>\nu$, and hence $|h(\mathbf{x})|>\nu / 2$. Then $|h|_{L^{p}(\Omega)^{l}} \geq \frac{\nu}{2}\left|\Omega_{\nu}^{-}(h)\right|^{1 / p}$, and consequently

$$
\lim _{|h|_{L^{p}(\Omega)^{l}} \rightarrow 0}\left|\Omega_{\nu}^{-}(h)\right|=0 .
$$

For all $\mathbf{x} \in \Omega$, we have that $|b(u+h)(\mathbf{x})| \leq|(u+h)(\mathbf{x})|$ and (when $|u+h|,|u|>0)$

$$
\begin{aligned}
q(u+h) & -q(u)-Q(u+h) h \\
& =-\frac{u}{|u+h||u|}\left(|u+h|-|u|-\frac{(u+h)^{\mathrm{T}} h}{|u+h|}\right)+\frac{(u+h)^{\mathrm{T}} h}{|u+h|^{2}}\left(\frac{u+h}{|u+h|}-\frac{u}{|u|}\right) .
\end{aligned}
$$

First, suppose that $\mathbf{x} \in \Omega_{\nu}^{+}(h)$. Then, we have $|u(\mathbf{x})|>\nu / 2$ and $|(u+h)(\mathbf{x})|>\nu$ and observe that

$$
\begin{aligned}
& |b(u+h)(\mathbf{x})(q(u+h)-q(u)-Q(u+h) h)(\mathbf{x})| \\
& \leq\left|\left(|u+h|-|u|-\frac{(u+h)^{\mathrm{T}} h}{|u+h|}\right)(\mathbf{x})\right|+\frac{2|h(\mathbf{x})|}{\nu^{2}}|(u|u|-u|u+h|+|u| h)(\mathbf{x})| \\
& \leq\left|\left(|u+h|-|u|-\frac{(u+h)^{\mathrm{T}} h}{|u+h|}\right)(\mathbf{x})\right|+\frac{4}{\nu^{2}}|u(\mathbf{x})||h(\mathbf{x})|^{2} .
\end{aligned}
$$

Second, suppose that $\mathbf{x} \in \Omega_{\nu}^{-}(h)$. In this case, we have $|u(\mathbf{x})| \leq \nu / 2$ and $|(u+h)(\mathbf{x})|>$ $\nu$ and observe that

$$
\begin{aligned}
& |b(u+h)(\mathbf{x})(q(u+h)-q(u)-Q(u+h) h)(\mathbf{x})| \\
& \leq\left|\left(|u+h|-|u|-\frac{(u+h)^{\mathrm{T}} h}{|u+h|}\right)(\mathbf{x})\right|+2|h(\mathbf{x})| .
\end{aligned}
$$

Hölder's inequality implies (because $3 \leq 3 s \leq p$ ) that

$$
\begin{aligned}
& |b(u+h)(q(u+h)-q(u)-Q(u+h) h)|_{L^{s}\left(\Omega_{\nu}(h)\right)^{l}} \\
& \leq|| u+h|-| u\left|-\frac{(u+h)^{\mathrm{T}} h}{|u+h|}\right|_{L^{s}(\Omega)} \\
& \quad+\frac{4 \beta(|\Omega|)}{\nu^{2}}|u|_{L^{p}(\Omega)^{l}}|h|_{L^{p}(\Omega)^{l}}^{2}+2\left|\Omega_{\nu}^{-}(h)\right|^{(p-s) /(p s)}|h|_{L^{p}(\Omega)^{l}},
\end{aligned}
$$

Copyright (c) by SIAM. Unauthorized reproduction of this article is prohibited. 
with $\beta(|\Omega|)=1$ if $3 s=p$ and $\beta(|\Omega|)=|\Omega|^{(p-3 s) / p s}$ if $3 s<p$.

Then, since $u \mapsto \frac{u^{\mathrm{T}}}{|u|}$ is a Newton derivative of $|\cdot|: L^{p}(\Omega)^{l} \rightarrow L^{s}(\Omega)$ when $u(\mathbf{x}) \neq 0$ and $\lim _{|h|_{L^{p}(\Omega)^{l}} \rightarrow 0}\left|\Omega_{\nu}^{-}(h)\right|=0$, we observe that

$$
I=o\left(|h|_{L^{p}(\Omega)^{l}}\right) .
$$

Now we turn our attention to $I I$. We define the following disjoint partition of $\Omega=\cup_{i=1}^{4} \Omega^{i}(h)$ :

$$
\begin{aligned}
& \Omega^{1}=\{\mathbf{x} \in \Omega:|(u+h)(\mathbf{x})|>\nu / 2 \text { and }|u(\mathbf{x})|>\nu / 4\} \\
& \Omega^{2}=\{\mathbf{x} \in \Omega:|(u+h)(\mathbf{x})|>\nu / 2 \text { and }|u(\mathbf{x})| \leq \nu / 4\} \\
& \Omega^{3}=\{\mathbf{x} \in \Omega:|(u+h)(\mathbf{x})| \leq \nu / 2 \text { and }|u(\mathbf{x})|>3 \nu / 4\} \\
& \Omega^{4}=\{\mathbf{x} \in \Omega:|(u+h)(\mathbf{x})| \leq \nu / 2 \text { and }|u(\mathbf{x})| \leq 3 \nu / 4 v\} .
\end{aligned}
$$

If $\mathbf{x} \in \Omega^{4}$, then $b(u+h)-b(u)=0$, and hence $|I I|_{L^{s}(\Omega)^{l}} \leq|I I|_{L^{s}\left(\Omega^{1}\right)^{l}}+$ $|I I|_{L^{s}\left(\Omega^{2} \cup \Omega^{3}\right)^{l}}$. If $\mathbf{x} \in \Omega^{2} \cup \Omega^{3}$, then $|h(\mathbf{x})| \geq \nu / 4$. Therefore, we find $|h|_{L^{p}(\Omega)^{l}} \geq$ $\frac{\nu}{4}\left|\left(\Omega^{2} \cup \Omega^{3}\right)(h)\right|^{1 / p}$ and

$$
\lim _{|h|_{L^{p}(\Omega)^{l}} \rightarrow 0}\left|\left(\Omega^{2} \cup \Omega^{3}\right)(h)\right|=0 .
$$

We know that $|(q(u+h)-q(u))(\mathbf{x})| \leq 2$, and therefore

$$
\begin{aligned}
|I I|_{L^{s}\left(\Omega^{2} \cup \Omega^{3}\right)^{l}} & \leq 2|b(u+h)-b(u)|_{L^{s}\left(\Omega^{2} \cup \Omega^{3}\right)^{l}} \leq 2|h|_{L^{s}\left(\Omega^{2} \cup \Omega^{3}\right)^{l}} \\
& \leq 2\left|\left(\Omega^{2} \cup \Omega^{3}\right)(h)\right|^{\frac{p-s}{p s}}|h|_{L^{p}(\Omega)^{l}},
\end{aligned}
$$

implying $|I I|_{L^{s}\left(\Omega^{2} \cup \Omega^{3}\right)^{l}}=o\left(|h|_{L^{p}(\Omega)^{l}}\right)$.

If $\mathbf{x} \in \Omega^{1}$, then from a simple calculation we observe

$$
|(q(u+h)-q(u))(\mathbf{x})| \leq \frac{16}{\nu^{2}}|u(\mathbf{x})||h(\mathbf{x})|,
$$

which implies by Hölder's inequality (note $3 \leq 3 s \leq p$ ) that

$$
\begin{aligned}
\mid(q(u)-q(u+h))(b(u+h) & -b(u))\left.\right|_{L^{s}\left(\Omega^{1}\right)^{l}} \\
& \leq \frac{16}{\nu^{2}}\left|u h^{2}\right|_{L^{s}(\Omega)^{l}} \leq \frac{16 \beta(|\Omega|)}{\nu^{2}}|u|_{L^{p}(\Omega)^{l}}|h|_{L^{p}(\Omega)^{l}}^{2},
\end{aligned}
$$

with $\beta(|\Omega|)=1$ if $3 s=p$ and $\beta(|\Omega|)=|\Omega|^{(p-3 s) / p s}$ if $3 s<p$. Then, $|I I|_{L^{s}\left(\Omega^{1}\right)^{l}}=$ $O\left(|h|_{L^{p}(\Omega)^{l}}^{2}\right)$. We conclude that

$$
I I=o\left(|h|_{L^{p}(\Omega)^{l}}\right) .
$$

Finally, it is simple to argue that

$$
I I I=o\left(|h|_{L^{p}(\Omega)^{l}}\right),
$$

since $q$ is bounded.

Theorem A.2 implies the following result.

Copyright $@$ ( ) by SIAM. Unauthorized reproduction of this article is prohibited. 
Corollary A.3. Let $W \equiv W_{0}^{1, p}(\Omega)$ and $X \equiv W_{0}^{1, s^{\prime}}(\Omega)$, where $\frac{1}{s}+\frac{1}{s^{\prime}}=1$ and $3 \leq 3 s \leq p<\infty$. Let $\mathfrak{P}: W \rightarrow X^{\prime}$ be

$$
(\mathfrak{P}(u), w)_{X^{\prime}, X}:=\int_{\Omega^{+}(u)} P(\nabla u) \cdot \nabla w \mathrm{~d} \mathbf{x}=\int_{\Omega^{+}(u)}(|\nabla u|-\varphi)^{+} \frac{(\nabla u \cdot \nabla w)}{|\nabla u|} \mathrm{d} \mathbf{x}
$$

for all $w \in X$ and where $P(\nabla u)=q(\nabla u) b(\nabla u)$. Then $G_{\mathfrak{P}}(u): W \rightarrow X^{\prime}$, given by

$$
\left(G_{\mathfrak{P}}(u) v, w\right)_{X^{\prime}, X}:=\int_{\Omega^{+}(u)}\left(G_{P}(\nabla u) \nabla v\right) \cdot \nabla w \mathrm{~d} \mathbf{x}
$$

for all $u, v \in W$ and $w \in X$, is a Newton derivative of $\mathfrak{P}$.

Proof. The map $\nabla: W_{0}^{1, p}(\Omega) \rightarrow L^{p}(\Omega)^{l}$ is Fréchet differentiable with $\nabla$ as its derivative, and $P$ is Newton differentiable as a mapping from $L^{p}(\Omega)^{l}$ to $L^{s}(\Omega)^{l}$ for $3 \leq 3 s \leq p$. Then $u \mapsto P(\nabla u)$ is Newton differentiable (because the composition of a Newton and a Fréchet differentiable mapping is Newton differentiable; see, e.g., [26]) as a mapping from $W_{0}^{1, p}(\Omega)$ to $L^{s}(\Omega)^{l}$ with a Newton derivative $u \mapsto G_{P}(\nabla u) \nabla$. Therefore, by Hölder's inequality,

$$
\begin{aligned}
& \mid(\mathfrak{P}(u+h)-\left.\mathfrak{P}(u)-G_{\mathfrak{P}}(u+h) h, w\right)_{X^{\prime}, X} \mid \\
& \quad \leq \int_{\Omega^{+}(u)}\left|P(\nabla(u+h))-P(\nabla u)-G_{P}(\nabla(u+h)) \nabla h\right||\nabla w| \mathrm{d} \mathbf{x} \\
& \quad \leq\left|P(\nabla(u+h))-P(\nabla u)-G_{P}(\nabla(u+h)) \nabla h\right|_{L^{s}(\Omega)^{l}}|\nabla w|_{L^{s^{\prime}}(\Omega)^{l}} .
\end{aligned}
$$

Since $\Omega$ is a bounded domain, then $|\nabla w|_{L^{s^{\prime}}(\Omega)^{l}}=|w|_{X}$ (where $X=W_{0}^{1, s^{\prime}}(\Omega)$ ) for $1 \leq s^{\prime} \leq \infty$ (because the Poincaré inequality holds even in the case $s^{\prime}=\infty$ when $|\Omega|<\infty$; see [44]). Therefore

$$
\begin{aligned}
\mid \mathfrak{P}(u+h)-\mathfrak{P}(u)-G_{\mathfrak{P}} & \left.(u+h) h\right|_{X^{\prime}} \\
& \leq\left|P(\nabla(u+h))-P(\nabla u)-G_{P}(\nabla(u+h)) \nabla h\right|_{L^{s}(\Omega)^{l}} .
\end{aligned}
$$

Since the right-hand side goes to zero as $|h|_{W} \rightarrow 0$, the statement is proved.

Appendix B. Conditions for $u \mapsto S(A, f, \Phi(u))$ to be contractive. Throughout this appendix, we denote the norm of $W_{0}^{1, p}(\Omega)$ as $|\cdot|_{W_{0}^{1, p}(\Omega)}\left(\right.$ instead of $\left.|\cdot|_{W}\right)$. For the sake of brevity, we will denote $S(f, \Phi(u)) \equiv S(A, f, \Phi(u))$, assume $\Omega$ is a bounded domain and $C_{p}$ denotes the Poincaré's constant associated to $W_{0}^{1, p}(\Omega)$, i.e., for all $v \in W_{0}^{1, p}(\Omega),|v|_{L^{p}(\Omega)} \leq C_{p}|v|_{W_{0}^{1, p}(\Omega)}$, where $C_{p}>0$ depends only on $p$ and $\Omega$.

Theorem B.1. Let $1<p<\infty$ and assume the following:

(i) $A: W_{0}^{1, p}(\Omega) \rightarrow W^{-1, p^{\prime}}(\Omega)$ satisfies $\mathbf{H 1}$ with $\min (2, p) \geq r>1$, H2, and $\mathbf{H 5}$ with $\beta \geq 1$.

(ii) $f \in L^{r^{\prime}}(\Omega) \subset W^{-1, p^{\prime}}(\Omega)$ such that $(f, v)=\int_{\Omega} f v \mathrm{~d} \mathbf{x}$ for all $v \in W_{0}^{1, p}(\Omega)$, where $1 / r+1 / r^{\prime}=1$ and $1 / p+1 / p^{\prime}=1$.

(iii) $\Phi: W_{0}^{1, p}(\Omega) \rightarrow L_{\nu}^{\infty}(\Omega) \subset L^{\infty}(\Omega)$ is defined as $\Phi(u)=\lambda(u) \phi$ with $\phi \in L^{\infty}(\Omega)$ such that $\phi(\mathbf{x})>0$ a.e. on $\Omega$, and the map $u \mapsto \lambda(u)$ satisfies the following: a. $\lambda: \bar{B}_{R}(0) \subset W_{0}^{1, p}(\Omega) \rightarrow \mathbb{R}^{+}$with $R:=\left(C_{p}|f|_{L^{r^{\prime}}(\Omega)}|\Omega|^{(p-r) / p}\right)^{1 /(r-1)}$, $\underline{\lambda} \leq \lambda(u) \leq \bar{\lambda}$ for all $u \in \bar{B}_{R}(0)$ and $0<\nu \leq \underline{\lambda} \phi(\mathbf{x})$ a.e. in $\Omega$.

b. $|\lambda(v)-\lambda(w)| \leq L_{\lambda}|v-w|_{W_{0}^{1, p}(\Omega)}$ for all $v, w \in \bar{B}_{R}(0)$ and some $L_{\lambda}>0$.

Copyright $@$ by SIAM. Unauthorized reproduction of this article is prohibited. 
Then, the map $u \mapsto S(f, \Phi(u))$ satisfies that $S(f, \Phi(\cdot)): \bar{B}_{R}(0) \rightarrow \bar{B}_{R}(0)$ and

$$
\left|S\left(f, \Phi\left(u_{1}\right)\right)-S\left(f, \Phi\left(u_{2}\right)\right)\right|_{W_{0}^{1, p}(\Omega)} \leq L_{S}(f)\left|u_{1}-u_{2}\right|_{W_{0}^{1, p}(\Omega)}
$$

for all $u_{1}, u_{2} \in \bar{B}_{R}(0)$ and some $L_{S}(f)>0$ such that $\lim _{|f|_{L^{r^{\prime}}(\Omega)} \rightarrow 0} L_{S}(f)=0$.

Proof. Let $\varphi \in L_{\nu}^{\infty}(\Omega), f_{1}, f_{2} \in L^{r^{\prime}}(\Omega) \subset W^{-1, p^{\prime}}(\Omega)$ such that $\left(f_{i}, v\right)=\int_{\Omega} f_{i} v \mathrm{~d} \mathbf{x}$ for $v \in W_{0}^{1, p}(\Omega)$. Let $u_{i}=S\left(f_{i}, \varphi\right)$ for $i=1,2$. Then, the uniform monotonicity implies

$$
c\left|u_{1}-u_{2}\right|_{W_{0}^{1, p}(\Omega)}^{r} \leq\left(A\left(u_{1}\right)-A\left(u_{2}\right), u_{1}-u_{2}\right) \leq\left(f_{1}-f_{2}, u_{2}-u_{1}\right) .
$$

Young's inequality states that $\int_{\Omega}|g v| \mathrm{d} \mathbf{x} \leq \frac{\epsilon^{r^{\prime}}}{r^{\prime}} \int_{\Omega}|g|^{r^{\prime}} \mathrm{d} \mathbf{x}+\frac{1}{r \epsilon^{r}} \int_{\Omega}|v|^{r} \mathrm{~d} \mathbf{x}$ for all $g \in$ $L^{r^{\prime}}(\Omega)$, all $v \in L^{r}(\Omega)$, and all $\epsilon>0$. Since $p \geq r$ by Young's, Hölder's (when $p>r$ ), and Poincaré's inequalities,

$$
\begin{aligned}
c\left|u_{1}-u_{2}\right|_{W_{0}^{1, p}(\Omega)}^{r} & \leq\left(f_{1}-f_{2}, u_{2}-u_{1}\right) \\
& \leq \frac{\epsilon^{r^{\prime}}}{r^{\prime}} \int_{\Omega}\left|f_{1}-f_{2}\right|^{r^{\prime}} \mathrm{d} \mathbf{x}+\frac{1}{r \epsilon^{r}} \int_{\Omega}\left|u_{1}-u_{2}\right|^{r} \mathrm{~d} \mathbf{x} \\
& \leq \frac{\epsilon^{r^{\prime}}}{r^{\prime}} \int_{\Omega}\left|f_{1}-f_{2}\right|^{r^{\prime}} \mathrm{d} \mathbf{x}+\frac{1}{r \epsilon^{r}}|\Omega|^{(p-r) / p}\left(\int_{\Omega}\left|u_{1}-u_{2}\right|^{p} \mathrm{~d} \mathbf{x}\right)^{r / p}, \\
& \leq \frac{\epsilon^{r^{\prime}}}{r^{\prime}} \int_{\Omega}\left|f_{1}-f_{2}\right|^{r^{\prime}} \mathrm{d} \mathbf{x}+\frac{C_{p}^{r}}{r \epsilon^{r}}|\Omega|^{(p-r) / p}\left(\int_{\Omega}\left|\nabla u_{1}-\nabla u_{2}\right|^{p} \mathrm{~d} \mathbf{x}\right)^{r / p} .
\end{aligned}
$$

Hence for a sufficiently large $\epsilon>0$,

$$
\left|u_{1}-u_{2}\right|_{W_{0}^{1, p}(\Omega)} \leq\left(\frac{\frac{\epsilon^{r^{\prime}}}{r^{\prime}}}{\left(c-\frac{C_{p}^{r}|\Omega|^{(p-r) / p}}{r \epsilon^{r}}\right)}\right)^{1 / p}\left(\int_{\Omega}\left|f_{1}-f_{2}\right|^{r^{\prime}} \mathrm{d} \mathbf{x}\right)^{1 / r} .
$$

Therefore, for $p \geq r>1$, we have $\Theta_{1}>0$ such that

$$
\left|S\left(f_{1}, \varphi\right)-S\left(f_{2}, \varphi\right)\right|_{W_{0}^{1, p}(\Omega)} \leq \Theta_{1}\left|f_{1}-f_{2}\right|_{L^{r^{\prime}}(\Omega)}^{r^{\prime} / r} .
$$

Suppose $\mu>0$ is such that $\mu \varphi \in L_{\nu}^{\infty}(\Omega)$. Since $A$ satisfies H5, $A(t u)=t^{\beta} A(u)$ for all $t>0$ and all $u \in W_{0}^{1, p}(\Omega)$. Thus, if $u=S(f, \varphi)$, then $\mu u=S\left(\mu^{\beta} f, \mu \varphi\right)$ (because $\mu>0)$ or, equivalently, $S(f, \varphi)-S\left(\mu^{\beta} f, \mu \varphi\right)=(1-\mu) S(f, \varphi)$. Using this and (B.1), we observe

$$
\begin{aligned}
\mid S(f, \varphi)- & \left.S(f, \mu \varphi)\right|_{W_{0}^{1, p}(\Omega)} \\
& \leq\left|S(f, \varphi)-S\left(\mu^{\beta} f, \mu \varphi\right)\right|_{W_{0}^{1, p}(\Omega)}+\left|S\left(\mu^{\beta} f, \mu \varphi\right)-S(f, \mu \varphi)\right|_{W_{0}^{1, p}(\Omega)} \\
& \leq|1-\mu||S(f, \varphi)|_{W_{0}^{1, p}(\Omega)}+\Theta_{1}\left|1-\mu^{\beta}\right|^{r^{\prime} / r}\left(\int_{\Omega}|f|^{r^{\prime}} \mathrm{d} \mathbf{x}\right)^{1 / r} \\
& =|1-\mu||S(f, \varphi)|_{W_{0}^{1, p}(\Omega)}+\Theta_{1}\left|1-\mu^{\beta}\right|^{1 /(r-1)}|f|_{L^{r^{\prime}}(\Omega)}^{r^{\prime} / r} .
\end{aligned}
$$

Suppose that $0<\mu \leq \bar{\mu}$ for some fixed $\bar{\mu}>0$. Since $2 \geq r>1$ and $\beta \geq 1$, it holds that $\left|1-\mu^{\beta}\right|^{1 /(r-1)} \leq \Theta_{2}|1-\mu|$ for some $\Theta_{2}>0$ (depending only on $\bar{\mu}$ ) for all $\mu \in(0, \bar{\mu}]$.

Copyright (c) by SIAM. Unauthorized reproduction of this article is prohibited. 
Since $|1-\mu| \nu \leq|\varphi-\mu \varphi|_{L^{\infty}(\Omega)}$ because $\varphi \in L_{\nu}^{\infty}(\Omega)$, then from (B.2) we have

$$
\begin{aligned}
|S(f, \varphi)-S(f, \mu \varphi)|_{W_{0}^{1, p}(\Omega)} & \leq\left(|S(f, \varphi)|_{W_{0}^{1, p}(\Omega)}+\Theta_{1} \Theta_{2}|f|_{L^{r^{\prime}(\Omega)}}^{r^{\prime} / r}\right)|1-\mu| \\
& \leq\left(|S(f, \varphi)|_{W_{0}^{1, p}(\Omega)}+\Theta_{1} \Theta_{2}|f|_{L^{r^{\prime}}(\Omega)}^{r^{\prime} / r}\right) \nu^{-1}|\varphi-\mu \varphi|_{L^{\infty}(\Omega)} .
\end{aligned}
$$

Using $v=0$ in (4.1), the uniform monotonicity and the homogeneity of $A$ imply $|u|_{W_{0}^{1, p}(\Omega)}^{r} \leq(A(u), u) \leq(f, u)$. Hence,

$$
|S(f, \varphi)|_{W_{0}^{1, p}(\Omega)} \leq\left(C_{p}|\Omega|^{(p-r) / p}|f|_{L^{r^{\prime}}(\Omega)} / c\right)^{1 /(r-1)}=R .
$$

From (B.3), we obtain

$$
|S(f, \varphi)-S(f, \mu \varphi)|_{W_{0}^{1, p}(\Omega)} \leq \Theta_{3}(f)|\varphi-\mu \varphi|_{L^{\infty}(\Omega)},
$$

for which $\lim _{|f|_{L^{r^{\prime}}(\Omega)} \rightarrow 0} \Theta_{3}(f)=0$.

Now, let $\varphi=\lambda\left(u_{1}\right) \phi, \mu:=\lambda\left(u_{2}\right) / \lambda\left(u_{1}\right)$ for some $u_{1}, u_{2} \in \bar{B}_{R}(0) \subset W_{0}^{1, p}(\Omega)$. This implies that $\mu \varphi=\lambda\left(u_{2}\right) \phi$. Since $\mu \leq \bar{\mu}:=\bar{\lambda} / \underline{\lambda}<\infty$, the above inequality implies

$$
\begin{aligned}
\left|S\left(f, \lambda\left(u_{1}\right) \phi\right)-S\left(f, \lambda\left(u_{2}\right) \phi\right)\right|_{W_{0}^{1, p}(\Omega)} & \leq \Theta_{3}(f)|\phi|_{L^{\infty}(\Omega)}\left|\lambda\left(u_{1}\right)-\lambda\left(u_{2}\right)\right| \\
& \leq \Theta_{3}(f)|\phi|_{L^{\infty}(\Omega)} L_{\lambda}\left|u_{1}-u_{2}\right|_{W_{0}^{1, p}(\Omega)} .
\end{aligned}
$$

We observe that (B.4) implies that $S\left(f, \Phi\left(\bar{B}_{R}(0)\right)\right) \subset \bar{B}_{R}(0)$. Finally, define $L_{S}(f):=$ $\Theta_{3}(f)|\phi|_{L^{\infty}(\Omega)} L_{\lambda}$. Then by (B.6) we observe $u \mapsto S(f, \Phi(u))$ is Lipschitz continuous (with Lipschitz constant less than one for small enough $|f|_{L^{r^{\prime}}(\Omega)}$ ) on $\bar{B}_{R}(0)$.

Example B.2. Consider $p=2$ and some domain $\Omega$, so that $W_{0}^{1,2} \equiv H_{0}^{1}(\Omega)$, and $A=-\Delta: H_{0}^{1}(\Omega) \rightarrow H^{-1}(\Omega)$, where $\Delta$ denotes the Laplacian. In this case, we have that $r=2$ (and hence consider $f \in L^{2}(\Omega)$ ) and that $\beta=1$. Consider $\Phi(u)=\lambda(u) \phi$, with $\phi \equiv 1$ and

$$
\lambda(u)=\int_{\Omega}|\nabla u| \mathrm{d} \mathbf{x}+\nu .
$$

Let $R:=C_{2}|f|_{L^{2}(\Omega)}$. Then, for $u \in \bar{B}_{R}(0), \lambda(u) \phi(\mathbf{x}) \geq \nu$ for all $\mathbf{x} \in \Omega$ and also

$$
|\lambda(v)-\lambda(w)| \leq|\Omega|^{1 / 2}|v-w|_{H_{0}^{1}(\Omega)} .
$$

Further, by Theorem B.1, for a small enough $f$ (in the $L^{2}(\Omega)$ sense), the sequential approximation $u_{n}=S\left(f, \Phi\left(u_{n-1}\right)\right)$ with $u_{0} \in \bar{B}_{R}(0)$ converges to a fixed point of $u \mapsto S(f, \Phi(u))$.

\section{REFERENCES}

[1] R. A. Adams and J. J. F. Fournier, Sobolev Spaces, 2nd ed., Academic Press, New York, 2003.

[2] P. M. Anselone, Collectively Compact Operator Approximation Theory and Applications to Integral Equations, Prentice-Hall, Englewood Cliffs, NJ, 1971.

[3] P. M. Anselone And R. Ansorge, Compactness principles in nonlinear operator approximation theory, Numer. Funct. Anal. Optim., 1 (1979), pp. 589-618.

Copyright $@$ by SIAM. Unauthorized reproduction of this article is prohibited. 
[4] J. Appell and M. P. Pera, Noncompactness principles in nonlinear operator approximation theory, Pacific J. Math., 115 (1984), pp. 13-31.

[5] J. Appell And P. P. Zabrejko, Nonlinear Superposition Operators, Cambridge University Press, Cambridge, UK, 1990.

[6] H. Attouch, G. Buttazzo, and G. Michaille, Variational Analysis in Sobolev and BV Spaces, MOS-SIAM Ser. Optim. 6, SIAM, Philadelphia, 2006.

[7] J.-P. Aubin and H. Frankowska, Set-Valued Analysis, Birkhäuser, Basel, 2009.

[8] C. BAIOCChI AND A. CAPELO, Variational and Quasivariational Inequalities, WileyInterscience, New York, 1984.

[9] A. Bensoussan And J.-L. Lions, Controle impulsionnel et inéquations quasi-variationnelles d'évolutions, C. R. Acad. Sci. Paris, 276 (1974), pp. 1333-1338.

[10] A. Bensoussan and J.-L. Lions, Impulse Control and Quasi-variational Inequalities, GauthierVillars, Paris, 1984.

[11] P. Beremlijski, J. Haslinger, M. KoČvara, and J. Outrata, Shape optimization in contact problems with Coulomb friction, SIAM J. Optim., 13 (2002), pp. 561-587.

[12] L. A. Caffarelli, The Obstacle Problem, Lezione Fermiane, Accademia Nazionale dei Lincei, Rome; Scuola Normale Superior, Pisa, 1998.

[13] J. Chabrowski, Variational Methods for Potential Operator Equations: With Applications to Nonlinear Elliptic Equations, De Gruyter, Berlin, 1997.

[14] G. Duvaut and J.-P. Lions, Les Inéquations en Mécanique et en Physique, Dunod, Paris, 1972.

[15] I. Ekeland And R. Temam, Convex Analysis and Variational Problems, North-Holland, Amsterdam; American Elsevier, New York, 1976.

[16] F. FACChinei And J.-S. PANG, Finite-Dimensional Variational Inequalities and Complementary Problems, Springer Ser. Oper. Res. 1, Springer, Berlin, 2003.

[17] A. Friedman, Variational Principles and Free-Boundary Problems, Wiley-Interscience, New York, 1982.

[18] R. Glowinski, Numerical Methods for Nonlinear Variational Problems, Springer-Verlag, Berlin, 1984.

[19] R. Glowinski, J.-L. Lions, And R. Trémolières, Numerical Analysis of Variational Inequalities, North-Holland, Amsterdam, 1981.

[20] P. T. HARKer, Generalized Nash games and quasi-variational inequalities, European J. Oper. Res., 54 (1991), pp. 81-94.

[21] M. Hintermüller And J. De los Reyes, A Duality-Based Semismooth Newton Framework for Solving Variational Inequalities of the Second Kind, IFB-Report 35 (01/2010), Institute of Mathematics and Scientific Computing, University of Graz, Graz, Austria, 2010.

[22] M. Hintermüller, K. Ito, And K. Kunisch, The primal-dual active set strategy as a semismooth Newton method, SIAM J. Optim., 13 (2003), pp. 865-888.

[23] M. Hintermüller AND K. Kunisch, Path-following methods for a class of constrained minimization problems in function space, SIAM J. Optim., 17 (2006), pp. 159-187.

[24] M. HintermüLler AND K. Kunisch, PDE-constrained optimization subject to pointwise constraints on the control, the state, and its derivative, SIAM J. Optim., 20 (2009), pp. 11331156 .

[25] R. H. W. Hoppe and R. Kornhuber, Adaptive multilevel methods for obstacle problems, SIAM J. Numer. Anal., 31 (1994), pp. 301-323.

[26] K. Ito and K. Kunisch, Lagrange Multiplier Approach to Variational Problems and Applications, Adv. Des. Control 15, SIAM, Philadelphia, 2008.

[27] C. T. Kelley, Iterative Methods for Linear and Nonlinear Equations, Frontiers Appl. Math. 16, SIAM, Philadelphia, 1995.

[28] D. Kinderlehrer and G. Stampacchia, An Introduction to Variational Inequalities and Their Applications, Classics Appl. Math. 31, SIAM, Philadelphia, 2000.

[29] R. Kornhuber, Monotone multigrid methods for elliptic variational inequalities. I. Numer. Math., 69 (1993), pp. 167-184.

[30] A. S. Kravchuk and P. J. NeittaAnmäki, Variational and Quasi-variational Inequalities in Mechanics, Springer, Berlin, 2007.

[31] M. Kunze And J. Rodrigues, An elliptic quasi-variational inequality with gradient constraints and some of its applications, Math. Methods Appl. Sci., 23 (2000), pp. 897-908.

[32] J.-L. Lions, Sur le côntrole optimal des systemes distribuées, Enseigne, 19 (1973), pp. 125-166.

[33] J.-L. Lions, Asymptotic behaviour of solutions of variational inequalities with highly oscillating coefficients, in Applications of Methods of Functional Analysis to Problems in Mechanics, Proc. Joint Symp. IUTAM/IMU, Lecture Notes in Math. 503, Springer, Berlin, 1975, pp. $30-55$.

Copyright (c) by SIAM. Unauthorized reproduction of this article is prohibited. 
[34] D. G. Luenberger, Optimization by Vector Space Methods, John Wiley, New York, 1969.

[35] B. S. Mordukhovich And J. V. Outrata, Coderivative analysis of quasi-variational inequalities with applications to stability and optimization, SIAM J. Optim., 18 (2007), pp. 389412.

[36] J. Outrata, M. Kocvara, And J. Zowe, Nonsmooth Approach to Optimization Problems with Equilibrium Constraints: Theory, Applications and Numerical Results, Kluwer Academic Publishers, Dordrecht, The Netherlands, 1998.

[37] J.-S. PANG AND M. FunUShima, Erratum: Quasi-variational inequalities, generalized Nash equilibria, and multi-leader-follower games, Comput. Manag. Sci., 6 (2009), pp. 373-375.

[38] R. Precup, Methods in Nonlinear Integral Equations, Springer, Berlin, 2002.

[39] L. Prigozhin, On the Bean critical-state model in superconductivity, European J. Appl. Math., 7 (1996), pp. 237-247.

[40] L. QI And D. Sun, A Survey of Some Nonsmooth Equations and Smoothing Newton Methods, Applied Mathematics Report AMR 98/10, School of Mathematics, University of New South Wales, Sydney, Australia, 1998.

[41] J. F. Rodrigues, Obstacle Problems in Mathematical Physics, North-Holland, Amsterdam, 1987.

[42] J. F. Rodrigues And L. SAntos, A parabolic quasi-variational inequality arising in a superconductivity model, Ann. Scuola Norm. Sup. Pisa Cl. Sci. (4), 29 (2000), pp. 153-169.

[43] L. Santos, Variational problems with non-constant gradient constraints, Port. Math. (N.S.), 59 (2002), pp. 205-248.

[44] L. TARTAR, An Introduction to Sobolev Spaces and Interpolation Spaces, Springer, Berlin, 2007.

[45] R. Wolf, Über lineare approximationsreguläre Operatoren, Math. Nachr., 59 (1974), pp. 325341.

[46] E. ZEIDLER, Nonlinear Functional Analysis and Applications, Vol. II/B: Nonlinear Monotone Operators, Springer-Verlag, Berlin, 1989.

Copyright $@$ by SIAM. Unauthorized reproduction of this article is prohibited. 